\title{
Le corps enseignant des écoles protestantes du Québec : portrait statistique (1900-1989)
}

\section{François Melançon et M'hammed Mellouki}

Volume 45, numéro 1, été 1991

URI : https://id.erudit.org/iderudit/304946ar

DOI : https://doi.org/10.7202/304946ar

Aller au sommaire du numéro

Éditeur(s)

Institut d'histoire de l'Amérique française

ISSN

0035-2357 (imprimé)

1492-1383 (numérique)

Découvrir la revue

Citer cet article

Melançon, F. \& Mellouki, M. (1991). Le corps enseignant des écoles protestantes du Québec : portrait statistique (1900-1989). Revue d'histoire de l'Amérique française, 45(1), 3-38. https://doi.org/10.7202/304946ar
Résumé de l'article

Cette étude du corps enseignant des écoles protestantes du Québec entend mettre en lumière, à travers une analyse statistique, certaines caractéristiques permettant de mieux saisir la place, le rôle et la fonction des enseignants dans la société québécoise. Elle dresse un portrait mouvant de ce corps enseignant qui commence, au tournant des années quarante, à mieux s'organiser et à mieux se structurer. Un accroissement quasi continu du nombre de ses membres et de la durée de leur formation, ainsi qu'une amélioration de leurs conditions salariales sont ainsi enregistrés. De plus, l'étude révèle la part grandissante occupée par les hommes au sein de ce groupe, tout comme la présence significative des femmes mariées sur le marché du travail enseignant. 


\title{
LE CORPS ENSEIGNANT DES ÉCOLES PROTESTANTES DU QUÉBEC: PORTRAIT STATISTIQUE (1900-1989) ${ }^{1}$
}

FRANÇOIS MELANÇON

Université de Paris I

M'HAMMED MELLOUKI

Département d'administration et politique scolaires

Université Laval

\section{RÉSUMÉ}

Cette étude du corps enseignant des écoles protestantes du Québec entend mettre en lumière, à travers une analyse statistique, certaines caractéristiques permettant de mieux saisir la place, le rôle et la fonction des enseignants dans la société québécoise. Elle dresse un portrait mouvant de ce corps enseignant qui commence, au toumant des années quarante, à mieux s'organiser et à mieux se structurer. Un accroissement quasi continu du nombre de ses membres et de la durée de leur formation, ainsi qu'une amélioration de leurs conditions salariales sont ainsi enregistrés. De plus, l'étude révèle la part grandissante occupée par les hommes au sein de ce groupe, tout comme la présence significative des femmes mariées sur le marché du travail enseignant.

\begin{abstract}
Using a statistical analysis, this study of the teachers of Québec's Protestant schools intends to underscore a number of characteristics that will help readers to better grasp the place, the role and the function of teachers in Québec society. The study sketches a changing portrait of teachers who in the forties began to become better organized and better structured. The almost continuous growth in the number of members and the length of their training together with an improvement in their salary conditions is recorded. In addition, the study reveals the growing place occupied by men in this group as well as the significant presence of married women in the teaching profession.
\end{abstract}

1 Le présent article a été réalisé dans le cadre d'une recherche subventionnée par le Conseil de recherches en sciences humaines du Canada (CRSHC). François Melançon détient une bourse de doctorat et M'hammed Mellouki une bourse du Canada octroyées par le CRSHC auquel ils expriment leur reconnaissance. Madame Nicole Dorion-Poussart a collaboré à la cueillette des données statistiques et documentaires de l'étude. 
L'historiographie du système scolaire québécois a souvent négligé d'accorder, dans son analyse, une part active au fonctionnement des écoles protestantes. Elle s'est plutôt attardée à comprendre et à expliquer les particularités du système scolaire des catholiques et à n'accorder au secteur protestant qu'un rôle instrumental, celui d'illustration de la différence. Pourtant, entre 1875 et 1961, le secteur protestant est un système scolaire à part entière, régi par des règles, des structures et des hiérarchies autonomes, indépendantes du secteur catholique. Même si sa clientèle ne représente jamais plus de $10 \%$ de la clientèle de l'ensemble du système scolaire québécois, ses structures ont eu une influence déterminante sur la réorganisation du système scolaire amorcée au début des années 1960.

Le personnel enseignant constitue une des pierres angulaires de l'architecture de ce système. Son nombre, la qualité de sa formation, son expérience de travail et sa condition salariale sont autant de facteurs permettant de mieux saisir sa spécificité, ainsi que sa transformation et son adaptation face aux conjonctures socio-économique et culturelle, internes et externes à la question scolaire. Il n'est pas question ici de brosser un tableau exhaustif du développement du corps enseignant des écoles protestantes au cours du $\mathrm{XX}^{\mathrm{e}}$ siècle. Il s'agit plutôt d'en dresser les contours démographiques et de poser les premiers jalons d'une étude socio-historique sur la place, le rôle et les fonctions des enseignants dans la société québécoise, à travers les transformations qu'elle subit. Dans la première partie, nous brosserons un tableau quantitatif de l'évolution des enseignants des écoles publiques protestantes afin de mieux comprendre les besoins auxquels ils répondent. Nous verrons, dans les parties suivantes, quelles modifications sont apportées à leur formation générale et professionnelle, et quelles sont leurs conditions salariales.

\section{LES EFFECTIFS SCOLAIRES}

Depuis 1915, les écoles du secteur protestant sont regroupées en trois catégories: les écoles élémentaires, offrant les sept premières années du programme d'études - auxquelles peut s'ajouter, généralement en milieu urbain, une année d'enseignement préscolaire (kindergarten); les écoles intermédiaires (ou écoles modèles, jusqu'en 1924), situées particulièrement en milieu rural, donnant les sept années du niveau élémentaire et les deux premières du niveau secondaire; et les high schools (ou écoles académiques, jusqu'en 1924), réunissant les onze ou douze années du cours d'études, soit les sept du niveau élémentaire et les quatre ou cinq du niveau secondaire. Ce découpage reste inchangé jusqu'au début des années 1960, époque où il se modifie au gré des réformes et de l'uniformisation proposées 
par la commission Parent et imposées par l'État québécois. Depuis lors, les écoles publiques protestantes et catholiques sont régies par un modèle commun qui comprend six années au primaire - auxquelles s'ajoutent une année de maternelle et la possibilité d'une septième année de rattrapage, - cinq années au secondaire et deux années préuniversitaires offertes par les CÉGEPS.

Si le découpage des niveaux d'enseignement ne varie pas avant les grandes réformes scolaires des années soixante, leurs structures subissent quant à elles des transformations significatives à la faveur, entre autres, de la tendance à la centralisation (consolidation) des écoles rurales qui s'amorce dès les premières années du $\mathrm{XX}^{\mathrm{e}}$ siècle. $\mathrm{Ce}$ mouvement à long terme cherche d'abord à rationaliser l'utilisation des bâtiments scolaires et du personnel enseignant afin de contourner les limites financières des commissions scolaires en régions éloignées - particulièrement affligées par l'émigration urbaine - et d'offrir aux élèves un enseignement plus varié et plus efficace, correspondant davantage aux nouvelles philosophies éducatives qui attachent une importance particulière à l'individu et au cheminement personnel des élèves. Au début, la centralisation vise essentiellement le regroupement d'écoles élémentaires d'une ou deux classes, situées dans des municipalités scolaires voisines, au sein d'un même bâtiment. À partir des années 1930, le mouvement se diversifie et s'étend aux écoles intermédiaires et aux high schools ruraux. La centralisation ne s'effectue désormais plus seulement de façon horizontale, c'est-à-dire entre écoles de même niveau, mais aussi verticalement puisque les écoles intermédiaires et les high schools tendent à absorber les écoles de niveau inférieur.

Le processus de centralisation se fait de façon progressive, les commissions scolaires et les municipalités hésitant, dans les premières années, à se départir d'une institution qui, quoique coûteuse, restait un lieu d'identité culturelle fort. De 1905 à 1946, 57 écoles centrales viennent remplacer 204 petites écoles élémentaires. Cependant, on trouve encore, en 1942, 376 de ces écoles à classe unique. Il faudra attendre dix ans pour voir ce nombre baisser à 120, et presque autant pour qu'il tombe à $38^{2}$. Les données du tableau 1 illustrent bien ce mouvement de centralisation des écoles et les efforts de rationalisation de l'administration scolaire. La clientèle et le corps enseignant croissent tandis que le nombre d'écoles diminue, ce qui entraîne une augmentation du nombre moyen d'élèves et d'enseignants par école. Du

2 Claude Quintin, «Évolution du système d'enseignement au Québec», Revue de l'Association canadienne d'éducation de langue française, 7,3 (mars 1978): 29; RSDIP (19601961): xiv. 
TABLEAU 1

Nombre d'écoles, d'élèves inscrits et d'enseignants dans le secteur protestant public

(1901-1989)

\begin{tabular}{lcccccc}
\hline Année & \multicolumn{2}{c}{ Écoles sous contrôle } & \multicolumn{2}{c}{ Élèves inscrits } & \multicolumn{2}{c}{ Enseignants } \\
\hline & $N$ & Indice & $N$ & Indice & $N$ & Indice \\
\cline { 2 - 7 } $1900-1901$ & 951 & 100 & 35407 & $100^{\mathrm{b}}$ & 1427 & 100 \\
$1910-1911$ & 1027 & 108 & 49099 & $139^{\mathrm{b}}$ & 1825 & 128 \\
$1920-1921$ & 769 & 81 & 66338 & 187 & $2189^{\mathrm{c}}$ & 153 \\
$1930-1931$ & 682 & 72 & 74662 & 211 & 2681 & 188 \\
$1934-1935$ & 643 & 68 & 76670 & 217 & $2705^{\mathrm{c}}$ & 190 \\
$1940-1941$ & 612 & 64 & 70038 & 198 & $2874^{\mathrm{c}}$ & 201 \\
$1944-1945$ & 519 & 55 & 63549 & 179 & 2587 & 181 \\
$1950-1951$ & 380 & 40 & 71211 & 201 & 2789 & 195 \\
$1954-1955$ & 308 & 32 & 92113 & 260 & 3363 & 236 \\
$1960-1961$ & 331 & 35 & 112467 & 318 & 4557 & 319 \\
$1964-1965$ & 332 & 35 & 126322 & 357 & 5654 & 396 \\
$1970-1971$ & 294 & 31 & 135290 & 382 & 5801 & 407 \\
$1974-1975$ & 271 & 28 & 129467 & 366 & - & - \\
$1980-1981$ & 267 & 28 & 92117 & 260 & 6336 & 444 \\
$1985-1986$ & - & - & 82048 & 232 & 5336 & 374 \\
$1988-1989$ & - & - & 84235 & 238 & 5557 & 389 \\
\hline
\end{tabular}

a Jusqu'en 1960-1961, comprend le personnel non enseignant et, entre 1981 et 1989, comprend les enseignants d'EDAA.

b Comprend également les élèves et les enseignants des écoles privées.

c Comprend également les enseignants des écoles privées.

Sources: RSIP 1900-1911 à 1960-1961: Statistiques de l'enseignement, 1964-1965, 1970-1971; R. Ferland, Les commissions scolaires. Onze années de statistiques sur les écoles et les clientèles 1970-1971 à 1980-1981, Québec, ministère de l'ÉducationDirection des études économiques et démographiques, 1982; ministère de l'Éducation, Personnel de la Commission scolaire (fichier PERCOS), 1980 à 1989; Communication privée, 22 février 1990, Jean-Pierre Dufort, Direction des études économiques et démographiques, ministère de l'Education, 1984 et 1988.

début du siècle au début des années 1980, cette augmentation est de près de $900 \%$ chez les élèves et de $1200 \%$ chez les enseignants.

Malgré ses bienfaits pédagogiques et administratifs, la centralisation des écoles en milieu rural entraîne, en contrepartie, des conséquences fâcheuses pour les communautés locales qui voient leur stabilité sociale ébranlée par le processus d'urbanisation et le déclin du monde rural que précipite ce processus ${ }^{3}$. L'école constituant un agent

3 Satadal Dasgupta, Rural Canada: Structures and Change (Lewiston, N. Y. et Queenston, Ont., E. Mellen Press, 1988), 165. 
actif dans les transformations de la société ${ }^{4}$, le temps passé par les jeunes ruraux à l'extérieur de leur communauté de résidence, dans des institutions dont les valeurs sont avant tout urbaines, affaiblit les liens qu'ils entretiennent avec elle 5 . Tous les contemporains ne partagent cependant pas cet avis quant aux effets néfastes du système d'éducation sur la culture rurale ${ }^{6}$, mais il n'en demeure pas moins que l'exode vers la ville est massif et s'accélère au cours des années 1920 et 1930, entraînant des répercussions importantes sur l'organisation scolaire, tant dans ses orientations idéologiques que dans ses structures. Tandis qu'en 1911 près de $70 \%$ du personnel enseignant œuvre en milieu rural, la proportion n'est plus en 1945 que de $22 \%$ et atteint à peine $6 \%$ au milieu des années $1960^{7}$. Du côté des écoles et des élèves, aucune statistique n'est disponible avant 1944-1945. Pour ce qui est du milieu géographique, toutefois, les données pour les périodes subséquentes indiquent un mouvement migratoire chez les élèves semblable à celui des enseignants. À la fin de la Deuxième Guerre mondiale, $18 \%$ des élèves sont inscrits dans des écoles rurales contre seulement 5\% vingt ans plus tard. Du côté des établissements d'enseignement, le déplacement est plus lent et moins prononcé puisqu'il nécessite des aménagements plus complexes. Encore au milieu des années 1940, plus de $70 \%$ des écoles sont localisées en milieu rural. Il faut attendre les années 1950 et 1960 pour voir leur proportion se rapprocher de celle du personnel enseignant et de la clientèle scolaire ${ }^{8}$.

4 Voir à ce sujet, André Petitat, Production de l'école-Production de la société (Paris et Genève, Librairie Droz, 1982), 532 p.

5 Verner Smitheram, «School Consolidation on P.E.I.: a Mixed Blessing», Abegneit Review, 1: 69-70, cité par S. Dasgupta, op. cit., 165; voir aussi Anne Drummond, «Rural Depopulation, Teacher Certification and the Course of Study in Quebec Protestant Superior Schools, 1885-1915», communication présentée à la Canadian History of Education Association, 1982, 21, citée par Ronald Rudin, Histoire du Québec anglophone, 1759-1980 (Québec, Institut québécois de recherche sur la culture, 1986), 258.

6 Sur la position du directeur de l'enseignement protestant, voir Pervical, Life in School (s.l., n.é., 1940), 101; et sur les prises de positions de la presse rurale, voir N. H. Mair, Protestant Education in Québec (Québec, Conseil supérieur de l'éducation, 1981), 94-95. Voir également au sujet des querelles qui opposent la population des milieux ruraux, par l'intermédiaire de certains de ses journalistes comme Robert Sellar, aux membres du comité protestant du Conseil de l'Instruction publique, Rudin, op. cit., 253-259; Robert Sellar, The Tragedy of Quebec (Toronto, 1974); Robert Hill, Robert Sellar and the Huntingdon Gleaner: the Conscience of Rural Protestant Quebec, 1863-1919, thèse de Ph.D., Université McGill, 1970.

7 Un déplacement démographique semblable est également constaté au niveau des inscriptions à l'école normale Macdonald, fondée initialement en vue d'améliorer l'éducation de la population rurale; voir «Rapport du directeur de l'école normale Macdonald», RSDIP (19201921): 244; RSDIP (1944-1945): 240.

8 RSDIP (1910-1911): xxii; RSDIP (1960-1961): 254; Statistiques de l'enseignement (1944-1945): 209, 213, 219, 223; Statistiques de l'enseignement (1964-1965): 253. 
Quatre phases de transformation de la clientèle scolaire peuvent être identifiées depuis le début du siècle jusqu'à la fin des années 1980 (tableau 1). La première, influencée par l'intégration des enfants de la nouvelle communauté juive de Montréal au réseau scolaire protestant, s'étend sur les trente premières années du siècle; elle est caractérisée par le doublement des inscriptions dans l'ensemble des écoles. Par la suite, entre 1930 et 1945, on enregistre un mouvement à la baisse (15\%) attribuable, entre autres, aux répercussions de la crise économique et de la guerre sur le taux de natalité et d'immigration, ainsi que sur le travail en milieu agricole et maritime; cette diminution frappe principalement les écoles élémentaires et intermédiaires. Elle est suivie d'une reprise soutenue du mouvement de croissance entraînée par la hausse des inscriptions dans l'enseignement secondaire. Entre 1945 et 1971 , cette hausse est de près de $280 \%$ comparativement à $56 \%$ dans les classes de niveau élémentaire. ${ }^{9}$ Cette deuxième poussée de croissance s'explique, bien sûr, par la reprise de la natalité ainsi que par l'industrialisation et la modernisation du Québec au sortir de la Deuxième Guerre mondiale, mais elle doit aussi beaucoup à la reprise de l'immigration et à l'intégration massive des immigrants au réseau scolaire protestant ${ }^{10}$.

Par la suite, l'application de nouvelles dispositions législatives restreignant le choix de la langue d'enseignement - malgré les efforts déployés pour s'y adapter ${ }^{11}$, - jumelée à une faible croissance des taux de natalité et à l'émigration hors-Québec d'un certain nombre de familles anglophones ${ }^{12}$, provoque une nouvelle phase de décroissance

9 RSDIP (1944-1945): 200; R. Ferland, Les commissions scolaires. Onze années de statistiques sur les écoles et les clientèles 1970-1971 à 1980-1981 (Québec, Ministère de l'Éducation - Direction des études économiques et démographiques, 1982), 566.

10 À titre d'exemple, une étude de Claude Quintin sur le choix de la langue d'enseignement de la part des immigrants québécois, au tout début des années 1970, indique que $85 \%$ d'entre eux choisissent des établissements d'enseignement anglophones contre $15 \%$ des établissements francophones; Quintin, loc. cit., 45.

11 Selon les données compilées par la Direction des études économiques et démographiques du ministère de l'Éducation, le pourcentage des élèves des écoles publiques protestantes fréquentant une institution de langue française est passé de 1\% en 1970-1971 à 27\% en 1988-1989. Sur l'expansion du réseau des écoles protestantes de langue française, voir Dominique Clift et S. McLeod-Arnopoulos, "L'idéologie anglo-protestante et le système scolaire», Le fait anglais au Québec (Montréal, Libre Expression, 1979), 123; et sur l'adaptation de certaines commissions scolaires protestantes à la nouvelle législation linguistique, voir Susan Schachter, Working Papers on English Language Institutions in Quebec (Montréal, Alliance Québec, mars 1982), 88-176.

12 Une étude réalisée par G. Caldwell sur l'émigration hors-Québec des anglophones québécois révèle que 33\% des diplômés des écoles secondaires anglophones du Québec dont la langue maternelle est l'anglais quittent la province au cours des cinq années suivant la fin de leurs études. Par contre, ce taux n'est que de $19 \%$ chez ceux dont la langue maternelle est autre que l'anglais. Citée par Clift et Arnopoulos, op. cit., 121-122. 
du nombre d'inscriptions dans les écoles protestantes publiques, plus forte que la première. C'est ainsi qu'entre 1971 et 1986, le nombre d'élèves passe de 135290 à 82 048, soit une diminution de près de $40 \%$.

\section{Le personnel enseignant}

Avant la réforme des années 1960, le personnel enseignant des écoles protestantes publiques, à la différence de celui du secteur catholique, est composé presque exclusivement de laïcs et particulièrement de laïcs de foi protestante attestée. Les enseignants de religion juive, à la faveur de l'importante vague d'immigration de leurs coreligionnaires au tournant du siècle dernier et dans le premier quart du $\mathrm{XX}^{\mathrm{e}}$, sont les seuls à pouvoir échapper à cette règle. On les retrouve généralement sur l'île de Montréal, dans les écoles fréquentées en majorité par les enfants juifs ${ }^{13}$. Au cours des années 1950, un seul enseignant catholique est engagé - à titre exceptionnel soulignait alors le comité protestant - tandis que des candidats de foi islamique et hindoue se voient refuser le même droit ${ }^{14}$. Cette homogénéité confessionnelle du corps enseignant protestant va à l'encontre du mythe longtemps entretenu quant à sa diversité, par rapport au corps enseignant catholique. Il est bien vrai que les enseignants du secteur protestant n'appartiennent pas tous à la même Église, mais c'est là une des bases du système scolaire protestant au Québec: il est confessionnel sans être lié à une secte en particulier. Hormis les enseignants d'origine juive dont la présence est surtout imposée par la part importante occupée par les jeunes Juifs dans les classes du secteur protestant — près de $40 \%$ dans l'île de Montréal au cours des années vingt ${ }^{15}$, les membres de confession religieuse autre que protestante ne pouvaient se faire admettre au sein du personnel enseignant de ce secteur.

Le nouveau comité protestant, constitué à la suite de la réorganisation du système scolaire québécois, assouplit toutefois sa position sous les pressions répétées de la Provincial Association of Protestant Teachers (PAPT). Il l'adapte aux nouvelles réalités administrative, culturelle et démographique des années 1960. Afin de pallier les craintes d'une absorption au sein d'un système scolaire unifié et de

13 Sur la question de la présence des Juifs dans les écoles protestantes, voir R. Rudin, Histoire du Québec anglophone, 1759-1980 (Québec, Institut québécois de recherche sur la culture, 1986), 250-253; D. Rome, «Les Juifs dans le Québec anglophone», G. Caldwell et É. Waddell, Les anglophones du Québec de majoritaires à minoritaires (Québec, Institut québécois de recherche sur la culture, 1982), 173-177; Mair, op. cit., 45-50.

14 RSDIP (1959-1960): 44-45.

15 Ronald Rudin, op. cit., 251. 
laisser plus de place aux groupes ethniques dont la proportion se fait de plus en plus grande parmi la population des écoles protestantes, la première version (1967) des règlements régissant le caractère confessionnel des établissements d'enseignement protestants laisse une porte ouverte aux enseignants des autres confessions religieuses. Bien qu'elle stipule que «tout enseignant doit être de religion protestante ou juive», elle concède néanmoins qu'un enseignant ne remplissant pas ces conditions peut être engagé pour enseigner des matières autres que la religion «quand un tel engagement est estimé avantageux pour les élèves». La version de 1969 va même plus loin. Elle indique que «la compétence professionnelle [des enseignants] doit être considérée comme étant plus importante que le statut confessionnel» ${ }^{16}$. Ce rapport entre compétence professionnelle et appartenance religieuse est maintenu malgré la profonde réforme des règlements du comité protestant au milieu des années 1970, qui fait disparaître toute référence à une religion particulière pour l'engagement des enseignants. On ne demande plus dorénavant à l'enseignant engagé d'être de religion protestante ou juive, mais de respecter la philosophie et le caractère confessionnel des établissements protestants ${ }^{17}$. Ces amendements et ces modifications aux règlements du comité protestant ont des répercussions notables sur la composition confessionnelle du personnel enseignant des écoles protestantes. En 1988, par exemple, la proportion des enseignants de foi catholique travaillant dans les écoles protestantes est évaluée à près de $25 \%$ tandis que la présence d'enseignants musulmans, hindous, agnostiques et athées est signalée ${ }^{18}$.

Contrairement à sa composition confessionnelle, on sait peu de chose de la composition ethnique du corps enseignant protestant. Jusque dans les années 1960, mis à part les quelques Juifs d'origine russe, polonaise et allemande, il se compose presque exclusivement d'Anglo-Saxons. L'analyse du lieu de formation des enseignants du secteur protestant par Frances Crook, en 1962, est en ce sens révélatrice. Cette analyse montre que $66 \%$ des enseignants sont formés au Québec, $17 \%$ dans les différentes provinces canadiennes, 9\% dans les pays du Commonwealth et $2 \%$ aux États-Unis et en Amérique latine ${ }^{19}$. Donc aucun enseignant du vieux continent ou à

16 Comité protestant, Règlements du comité protestant du Conseil supérieur de l'éducation (Québec, CSE, janvier 1969), règlement no 3, article 4.

17 Comité protestant, Règlements du comité protestant du Conseil supérieur de l'éducation (Québec, CSE, septembre 1975), 14; Comité protestant, Règlements du comité protestant du Conseil supérieur de l'Éducation (Québec, CSE, 1988), 3.

18 Association provinciale des enseignants protestants du Québec, Mémoire présenté à la commission de l'éducation sur le projet de loi 107, loi sur l'instruction publique (s.1., n.é., avril 1988), 1.

19 F. Crook, The Teacher in the Protestant Schools of Quebec (miméographié, 1962), 30. 
peine quelques-uns. Par la suite, les transformations ethno-confessionnelles de la clientèle scolaire et du personnel enseignant, ainsi que les modifications législatives touchant la langue d'enseignement ont favorisé une présence de plus en plus grande d'enseignants francoquébécois ou provenant des communautés ethniques. Dans ce dernier cas, les incursions demeurent timides. Par exemple, selon les chiffres rapportés par Stephanos Constantinides, tandis que 8000 jeunes Québécois d'origine grecque fréquentent les écoles protestantes en 1980, seulement 120 membres de cette communauté font partie du corps enseignant ${ }^{20}$.

\section{Recrutement et nombre}

De façon générale, le nombre d'enseignants dans les écoles protestantes est en constante progression entre 1901 et 1981 . Cette progression est ponctuée de deux phases accélérées dans le premier tiers du siècle et depuis le début des années 1950 jusqu'au milieu des années 1960, entrecoupées d'une phase de croissance modérée (tableau 1).

Bien que laissant paraître des réactions différentes aux crises socio-économiques de 1930 et 1940 , les courbes du nombre d'élèves et d'enseignants font bien ressortir l'étroite relation entre clientèle scolaire et personnel enseignant, du moins jusque dans les années 1960 (graphique 1). Les périodes de croissance accélérée indiquées par les deux courbes correspondent en effet aux périodes de forte immigration et de natalité élevée au Québec. Ces courbes font aussi apparaître, après 1965, un corps enseignant dont la progression est peut-être moins déterminée par celle de la clientèle scolaire que par l'acquisition de la sécurité d'emploi au cours des négociations de conventions collectives de la fin des années 1960 et du début des années 1970.

Les courbes du nombre d'élèves et d'enseignants laissent également entrevoir que la réforme scolaire des années 1960 a joué un rôle limité dans la croissance démographique des effectifs scolaires. La réforme apparaît, en fait, davantage comme une réponse à cette croissance rapide enregistrée dans les années 1950 que comme un catalyseur. Tout au plus a-t-elle pu soutenir cette augmentation et proposer l'amélioration de l'administration de ces nouveaux effectifs. De fait, tandis que le corps enseignant augmente de $76 \%$ au cours des quinze années précédant 1961 , l'augmentation n'est que de près de $40 \%$ dans

20 S. Constantinides, Les Grecs du Québec. Analyse historique et sociologique (Montréal, Éditions O Metoikos-Le Métèque, 1983), 165-166. 


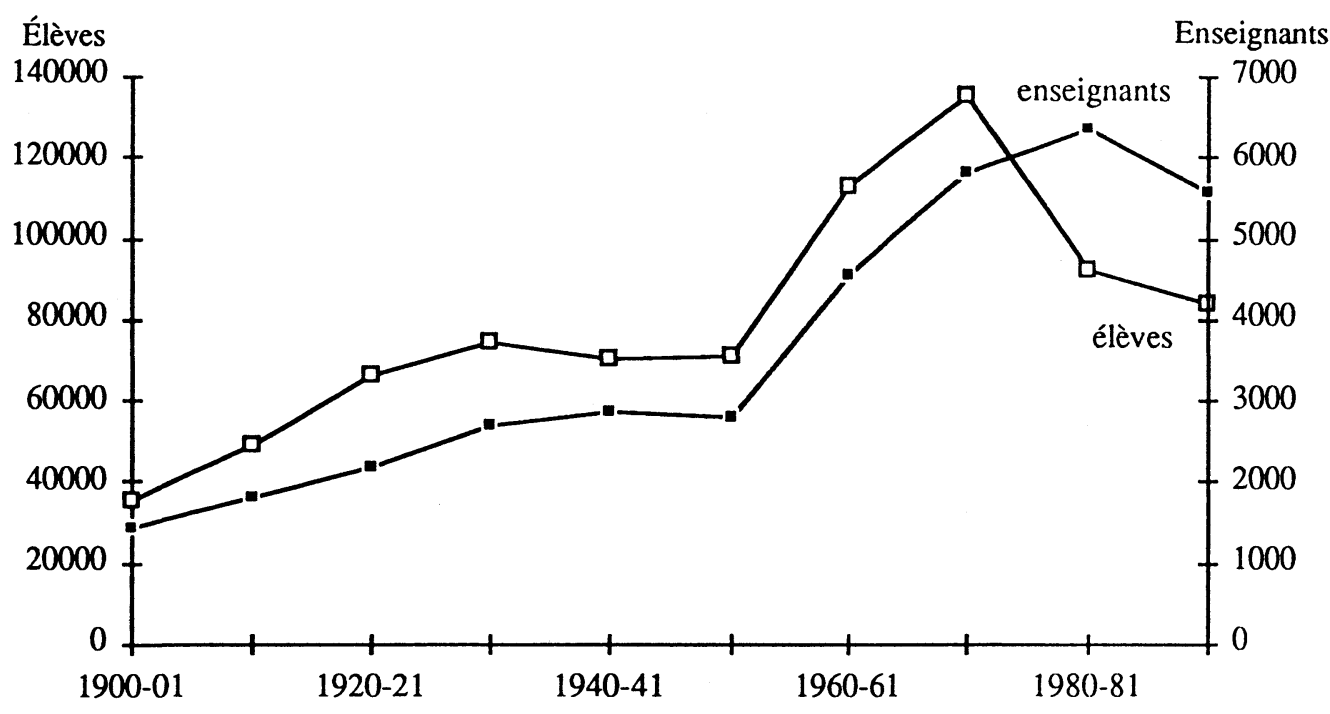

Sources: RSIP 1900-1911 à 1960-1961; Statistiques de l'enseignement 1970-1971; R. Ferland, Les commissions scolaires. Onze années de statistiques sur les écoles et les clientèles 1970-1971 à 19801981 (Québec, Ministère de l'Education - Direction des études économiques et démographiques, 1982); Ministère de l'Éducation, Personnel de la Commission scolaire (fichier PERCOS), 1980 à 1989; Communication privée, 22 février 1990, M. Jean-Pierre Dufort, Direction des études économiques et démographiques, Ministère de l'Éducation, 1984 et 1988. 
les vingt années subséquentes. Même écart dans le nombre des inscriptions, qui est cinq fois plus élevé dans les quinze années précédant 1961 que dans les quinze suivantes (tableau 1).

\section{TABLEAU 2}

Répartition en pourcentage des enseignantes et enseignants des écoles protestantes publiques selon le niveau d'enseignement

(1901-1989)

\begin{tabular}{lccccccccc}
\hline Année & \multicolumn{1}{c}{ Élémentaire } & \multicolumn{3}{c}{ Secondaire } & \multicolumn{4}{c}{ Ensemble $^{\mathbf{a}}$} \\
\hline & $\boldsymbol{H}$ & $\boldsymbol{F}$ & $\boldsymbol{H}$ & $\boldsymbol{F}$ & $\boldsymbol{H}$ & $\boldsymbol{F}$ & $\boldsymbol{N}$ & Indice \\
\cline { 2 - 9 } $1900-1901$ & 2 & 98 & 25 & 75 & 7 & 93 & 1 & 427 & 100 \\
$1920-1921$ & 3 & 97 & 20 & 80 & 8 & 92 & 2 & $189^{\mathrm{b}}$ & 153 \\
$1940-1941$ & 9 & 91 & 30 & 70 & 19 & 81 & 2 & $874^{\mathrm{b}}$ & 201 \\
$1960-1961$ & 9 & 91 & 50 & 50 & 24 & 76 & 4 & 557 & 319 \\
$1980-1981$ & 14 & 86 & 57 & 43 & 38 & 62 & 5541 & 388 \\
\hline
\end{tabular}

a Jusqu'en 1960-1961, comprend le personnel non enseignant. En 1981, les enseignants d'EDAA sont exclus des calculs parce qu'aucune répartition selon le niveau d'enseignement n'est effectuée dans les données du MEQ. Ils constituent, cette année-là, 795 enseignants supplémentaires.

b Comprend également les enseignants des écoles privées.

Sources: RSDIP 1900-1901 à 1960-1961; ministère de l'Éducation du Québec, Personnel de la commission scolaire (fichier PERCOS), 1980.

Ce personnel enseignant des écoles protestantes publiques est, tout au long de la période étudiée, majoritairement féminin (tableau 2). Toutefois, on y note un accroissement graduel de la proportion d'enseignants de sexe masculin, proportion qui passe de moins de $10 \%$ au début du siècle à plus de 35\% dans les années 1980 . Ce taux de croissance est près de onze fois supérieur à celui des femmes. Entre 1901, année de référence, et 1981, année où le personnel enseignant atteint son sommet, le nombre d'enseignants passe de 101 à 2289 $(+2189 \%)$, tandis que celui des enseignantes passe de 1326 à 4047 $(+205 \%)^{21}$. C'est surtout dans l'enseignement secondaire, secteur en plein développement après les années 1940, que se concentre la participation grandissante des enseignants de sexe masculin (tableau 2). Alors qu'ils ne forment jamais plus de $15 \%$ du personnel enseignant à l'élémentaire, ils constituent dès la fin des années 1950 - et peut-

21 RSDIP (1900-1901): xvii et xix; Ministère de l'Éducation du Québec, Personnel de la commission scolaire (fichier PERCOS), 1980. 
être même avant, compte tenu des changements survenus dans la tenue des statistiques officielles - plus de $50 \%$ du personnel enseignant au secondaire.

Plusieurs facteurs peuvent expliquer cette relative «masculinisation» du personnel enseignant dans le secteur protestant. Le principal est sans contredit l'attention accordée à cette question par les administrateurs de l'enseignement protestant, tant au sein du comité protestant que chez les membres de l'inspectorat, et aux efforts consentis par eux afin d'améliorer les conditions de travail des enseignants et de donner plus de prestige à la profession. C'est que la grande mobilité des enseignants masculins, ainsi que leur réticence à s'engager dans la profession enseignante, ont toujours inquiété les administrateurs scolaires. Ceux-ci ont régulièrement cherché à contrer ce mouvement et à trouver des solutions pour attirer et garder dans l'enseignement le personnel masculin. Aux yeux de P.W. Percival, directeur de l'enseignement protestant à la fin des années 1940, la meilleure façon est d'offrir des salaires plus équitables et comparables à ceux de certains secteurs de l'industrie, des possibilités de promotion adaptées, de s'assurer de la reconnaissance sociale de la profession et de changer la perception qu'en a le public en général. En effet, selon lui, l'enseignement est perçu à cette époque comme un emploi réservé aux femmes, quand ce n'est pas aux vieilles mégères ${ }^{22}$.

Mais ce n'est pas tout d'attirer des hommes dans la profession et de les garder, encore faut-il les garder en classe. Beaucoup d'enseignants masculins délaissent l'enseignement proprement dit et se laissent tenter par l'aventure de l'administration scolaire, généralement en raison des salaires qu'elle commande. En 1961, par exemple, plus de $40 \%$ des enseignants de sexe masculin possédant quinze années et plus d'expérience occupent un poste de personnel non enseignant: directeur d'école, orienteur, psychologue, directeur d'études, etc $^{23}$. Cette faille avait déjà été mise en évidence et critiquée par D. C. Munroe dans un article publié vers la fin des années cinquante ${ }^{24}$.

Notons finalement la croissance progressive du nombre d'enseignants dans les écoles secondaires (tableau 3). Continue jusqu'au début des années 1980, à l'exception de la diminution enregistrée au moment de la Deuxième Guerre mondiale, elle chute fortement $(21 \%)$ entre 1981 et 1986 pour se stabiliser à la fin de la décennie. Comparé

22 W. P. Percival, «A Few Developments in Education», The Educational Record, 63,2 (avril-juin 1947): 7.

23 RSDIP (1960-1961): 255.

24 D. C. Munroe, «Teacher Education and Teacher Shortage», The Teacher's Magazine, 37,185 (fév. 1957): 13. 
TABLEAU 3

Répartition en nombre et en pourcentage des enseignantes et enseignants des écoles protestantes publiques selon le niveau d'enseignement

(1901-1989)

\begin{tabular}{|c|c|c|c|c|c|c|c|c|}
\hline \multirow[t]{2}{*}{ Année } & \multicolumn{3}{|c|}{ Élémentaire } & \multicolumn{3}{|c|}{ Secondaire } & \multicolumn{2}{|c|}{ Ensemble } \\
\hline & $N$ & Indice & $\%$ & $N$ & Indice & $\%$ & $N$ & Indice \\
\hline $1900-1901$ & 1106 & 100 & 78 & 321 & 100 & 22 & 1427 & 100 \\
\hline $1910-1911$ & 1351 & 122 & 74 & 474 & 148 & 26 & 1825 & 128 \\
\hline $1920-1921$ & 1579 & 143 & 72 & 610 & 190 & 28 & 2189 & 153 \\
\hline $1930-1931$ & 1666 & 151 & 62 & 1015 & 316 & 38 & 2681 & 188 \\
\hline $1934-1935$ & 1658 & 150 & 61 & 1047 & 326 & 12 & 2705 & 190 \\
\hline $1940-1941$ & 1547 & 140 & 54 & 1327 & 413 & 46 & 2874 & 201 \\
\hline 1944-1945 & 1369 & 124 & 53 & 1218 & 379 & 47 & 2587 & 181 \\
\hline $1950-1951$ & 1411 & 128 & 51 & 1378 & 429 & 49 & 2789 & 195 \\
\hline $1954-1955$ & 1707 & 154 & 51 & 1656 & 516 & 49 & 3363 & 236 \\
\hline $1960-1961$ & 2835 & 256 & 62 & 1722 & 536 & 38 & 4557 & 319 \\
\hline $1964-1965$ & 3170 & 287 & 56 & 2484 & 774 & 44 & 5654 & 396 \\
\hline $1970-1971$ & 3285 & 297 & 57 & 2516 & 784 & 43 & 5801 & 407 \\
\hline $1974-1975$ & - & - & - & - & - & - & - & - \\
\hline $1980-1981^{a}$ & 2503 & 226 & 45 & 3038 & 946 & 55 & 5541 & 388 \\
\hline $1985-1986^{a}$ & 2310 & 209 & 49 & 2392 & 745 & 51 & 4702 & 330 \\
\hline $1988-1989^{a}$ & 2630 & 238 & 53 & 2304 & 718 & 47 & 4934 & 346 \\
\hline
\end{tabular}

a Le total exclut le personnel des classes de EDAA parce qu'aucune répartition selon le niveau d'enseignement n'est donnée dans les données du MEQ. Ils constituent respectivement 795, 634 et 623 enseignants supplémentaires.

Sources: RSIP 1900-1901 à 1960-1961; Statistiques de l'enseignement, 1964-1965, 1970-1971; ministère de l'Éducation du Québec, Personnel de la commission scolaire (fichier PERCOS), 1980, 1985, 1989.

au taux de croissance des enseignants du niveau élémentaire, celui des enseignants du niveau secondaire est près de 7 fois plus élevé. Entre 1901 et 1981 , il s'élevait à $846 \%$ contre $126 \%$ pour celui du niveau élémentaire. C'est ainsi que dans la première moitié des années 1980 le nombre des enseignants œuvrant au secondaire a surpassé celui de leurs collègues œuvrant à l'élémentaire.

\section{FORMATION ET QUALIFICATION}

La formation des enseignants est une préoccupation constante du comité protestant du Conseil de l'Instruction publique, qui doit faire face, surtout depuis les bouleversements socio-économiques des années 1930-1940 jusqu'au milieu des années 1970, à une pénurie chronique de personnel qualifié. Cette pénurie résulte principalement de la forte croissance de la clientèle scolaire et de l'insuffisance 
relative du nombre de nouveaux diplômés provenant des différentes institutions de formation. Leur nombre ne réussit jamais à assurer le renouvellement annuel du personnel. Tandis qu'on évalue à près de $15 \%$, au début des années soixante, le taux d'abandon de la profession, le nombre d'enseignants en formation ne représente, avant la fin des années cinquante, qu'environ 5\% du nombre des enseignants en exercice $^{25}$. Même chose au milieu des années 1970: on évalue à environ 1300 le nombre de nouveaux enseignants nécessaires annuellement pour assurer le fonctionnement des écoles anglophones de la province tandis que seulement 800 nouveaux enseignants diplômés sortent des universités McGill, Concordia et Bishop ${ }^{26}$.

\section{Les institutions de formation des enseignants}

La première école de formation professionnelle pour les enseignants des écoles protestantes ouvre ses portes en 1857, la même année que les deux premières écoles normales catholiques Laval et Jacques-Cartier ${ }^{27}$. Fondée par le directeur de l'Université McGill, John W. Dawson, la McGill Normal School est administrée par un comité où siègent des représentants des gouverneurs de l'Université McGill, du gouvernement provincial et du public en général. Ce comité est chargé du contrôle du budget, de l'engagement du personnel enseignant et de la définition du programme de l'institution. Jusqu'en 1907, année de son transfert au Collège Macdonald de Sainte-Anne-deBellevue, soixante élèves-maitres en moyenne fréquentent chaque année l'institution et plus de 4000 diplômes de niveaux maternelle, élémentaire, modèle et académique sont accordés ${ }^{28}$. Ces diplômes ne sont toutefois pas obligatoires pour enseigner, du moins pas avant 1900 , et l'émission des brevets d'enseignement relève, jusqu'en 1888, des bureaux d'examinateurs locaux. Ceux-ci sont chargés de faire passer aux candidats des examens de connaissances générales et pédagogiques et de délivrer les brevets, qui sont de trois catégories: élé-

25 Quebec Federation of Protestant Home and School Association, op. cit., 1962, 17.

26 D. A. Burgess, «Future Prospects for Education Graduates», McGill Journal of Education, 12,1 (print. 1977): 149-151.

27 Au sujet des institutions de formation professionnelle du secteur protestant, voir S. B. Frost, McGill University for the Advancement of Learning, II: 1895-1971 (Kingston et Montréal, McGill-Queen's University Press, 1984), 493 p.; J. D. Jefferies, «Bishop's University and the Training of Teachers», The Teacher's Magazine, 45,224 (nov. 1964): 32-34; D. C. Munroe, «The Education of Teachers Seventy Years Ago», The Educational Record, 67,1 (janv.-mars 1951): 13-19; D. C. Munroe, «Teacher Education at McGill», McGill Journal of Education, 6,1 (été 1971): 29-40; J. F. Snell, Macdonald College of McGill University. A History from 1904-1955 (Montréal, McGill University Press, 1963), 269 p.

28 Snell, op. cit. (1963), 238, note 5. 
mentaire, modèle et académique. Il existait onze de ces bureaux d'examinateurs locaux en 1888, époque où est mis en place un bureau central pour la province, le Protestant Central Board of Examiners (PCBE). À partir de 1889 , ce bureau central prend donc sous sa responsabilité les tâches jusque-là dévolues aux organismes locaux et voit graduellement à élever les critères de sélection des candidats pour assurer une meilleure qualité du personnel enseignant ${ }^{29}$. En 1896 , il juge insuffisants les examens comme critère de qualification et recommande qu'une formation professionnelle reconnue, acquise à l'université pour les candidats du brevet d'école académique, ou à l'École normale McGill pour les candidats des deux autres brevets, soit aussi prise en considération.

Deux ans plus tard, les nouveaux règlements du comité protestant viennent entériner cette décision du bureau central des examinateurs en le reconnaissant comme la seule instance pouvant admettre les candidats à l'école normale et décerner les diplômes d'enseignement pour les écoles protestantes du Québec ${ }^{30}$. Les examens sont désormais intégrés au programme de formation des enseignants et des pressions constantes sont exercées afin d'obliger les candidats et les enseignants sans formation à fréquenter l'université ou l'école normale ${ }^{31}$. Cependant, ce passage obligatoire n'a jamais pu être imposé rigoureusement, compte tenu de la pénurie chronique d'enseignants qu'a connue le secteur protestant. Mais depuis cette époque jusqu'à sa dissolution en 1970, la tâche du bureau central des examinateurs se modifie peu. Il voit, au cours de cette période, en collaboration avec le surintendant de l'Instruction publique (avant 1940), le directeur de l'enseignement protestant (de 1940 à 1964) ou le ministre de l'Éducation (après 1964) à l'émission des différents brevets d'enseignement.

En 1902 et 1903, à la suite des enquêtes de J. Adams et de G. H. Locke sur le système scolaire protestant et sur la formation de ses enseignants, la décision est prise de déménager à l'extérieur de Montréal la McGill Normal School et de l'intégrer au Collège Macdonald. Les liens qui relient alors l'école normale à l'Université McGill se voient modifier. Elle n'est désormais plus simplement

29 Sur l'histoire du Bureau central des examinateurs protestants, lire J. D. Jefferis, «Requiem for the Protestant Central Board of Examiners», McGill Journal of Education, 6,1 (print. 1971): 102-108.

30 Ibid., 104.

31 Dans l'«announcement» de la McGill Normal School de 1899-1900 se trouvent les propos suivants: «hereafter the Protestant Central Board of Examiners for the Province of Quebec will grant diplomas only to teachers-in-training of this institution and to graduates of British or Canadian Universities.» Cité par F. Crook, op. cit. (1962), 3. 
affiliée à cette dernière, mais directement placée sous son autorité ${ }^{32}$. L'université crée à cet effet à la Faculté des Arts et des Sciences un département d'éducation, en plus de présider le Teachers Training Committee (TTC) chargé de définir le programme d'études et les politiques de la nouvelle école normale. Les raisons de ce transfert et de l'intégration de l'école normale au sein de ce collège fondé principalement dans le but d'améliorer l'éducation de la population rurale $^{33}$ restent imprécises ${ }^{34}$.

En 1955, le département d'éducation et l'École normale Macdonald fusionnent pour donner naissance à l'Institut de pédagogie. Celuici demeure cependant intégré à la Faculté des Arts et des Sciences de l'Université McGill jusqu'à l'établissement, dix ans plus tard, d'une Faculté d'éducation proprement dite. Mil neuf cent soixante-dix marque finalement le dernier temps fort des transformations affectant les structures institutionnelles de formation des enseignants des écoles protestantes. À la suite de l'entente signée en décembre 1969 entre le Gouvernement québécois et l'Université McGill, tous les services offerts au Collège Macdonald sont transférés sur le campus universitaire montréalais, tandis que la formation des enseignants anglocatholiques, assurée jusque-là par le Saint Joseph Teachers' College de Montréal, est assumé par la Faculté de l'Éducation de l'Université McGill, dans un département conçu à cet effet ${ }^{35}$. Cette concentration des élèves-maîtres du secteur protestant sur le campus montréalais et leur cohabitation avec ceux du secteur anglo-catholique mettent un terme à l'isolationnisme qui caractérisait jadis la formation des enseignants du secteur protestant et constituent une étape de plus dans l'uniformisation de la formation de l'ensemble des maîtres du système scolaire québécois ${ }^{36}$.

32 En fait, comme le souligne N. H. Mair, c'est tout le collège Macdonald qui est placé sous l'autorité de McGill; Mair, op. cit. (1981), 5.

33 Outre l'école normale, le Collège Macdonald comptait également une école d'agriculture et une école des sciences ménagères. Sur le projet pédagogique de W. Macdonald, voir Frost, op. cit. (1984), 59-73.

34 Voir à ce sujet N. H. Mair, Protestant Education in Quebec. Notes on the History of Education in the Protestant Public Schools of Quebec (Québec, Comité protestant du Conseil supérieur de l'Éducation, 1981), 74, 94-100; Frost, op. cit. (1984), 64.

35 L' 'Agreement between the Government of Quebec and McGill University concerning teachers education in Montreal» est reproduit dans Gustave Crépeau, L'intégration de la formation et du perfectionnement des enseignants à l'Université McGill. Rapport présenté au Comité de la formation et du perfectionnement des enseignants, Commission d'étude sur les universités, 16 octobre 1978, 17.

36 Les universités Bishop et Concordia ont également joué un rôle actif dans la formation des enseignants des écoles protestantes, mais moins important, quantitativement. Tandis que la première maintient des activités soutenues de formation depuis 1898 auprès d'un petit nombre d'enseignants, la participation de la seconde ne débute qu'à la fin des années 1960 avec la création d'un département d'éducation. 


\section{Les brevets}

Le bureau central des examinateurs du secteur protestant, comme nous venons de le voir, décerne trois diplômes ou brevets dont les noms varient au cours de la période étudiée. Il s'agit du brevet élémentaire ou de classe III, du brevet modèle, ou intermédiaire, ou encore de classe II, ainsi que du brevet académique, ou de high schools, ou encore de classe $\mathrm{I}^{37}$. Légalement, le brevet n'est valide que pour le niveau qu'il indique ${ }^{38}$. Mais dans la réalité, compte tenu des difficultés de recrutement, un enseignant détenant un diplôme de classe III peut aussi bien enseigner au niveau secondaire qu'au niveau élémentaire, tout comme un enseignant détenant un diplôme de classe I ou de classe II peut enseigner au niveau élémentaire ou secondaire. Cependant, à partir de 1935 , le diplôme remis à la sortie de l'école normale n'est plus que temporaire et ne devient permanent qu'après deux années d'enseignement satisfaisant au cours des cinq ans suivant la sortie de l'école normale.

Les conditions nécessaires à l'obtention de ces diplômes sont modifiées plusieurs fois. L'élargissement des connaissances parmi la population, l'accroissement des savoirs en psychologie, en pédagogie et en sociologie, l'apparition de nouvelles technologies de l'enseignement (radio, télévision, films, etc.), la confrontation du niveau de formation des enseignants de la province avec celui des enseignants du reste du pays, rendue possible par les enquêtes pancanadiennes inaugurées en 1943, sont autant d'éléments qui jalonnent l'évolution de la formation des enseignants au cours de la période considérée, au point de vue de la valeur et du contenu. En 1934, la durée minimale de la formation professionnelle est définitivement fixée à un an. Pendant la même période, les cours d'été, à l'origine facultatifs, deviennent partie intégrante du programme de formation professionnelle des enseignants et constituent le premier pas vers un resserrement des conditions d'émission des diplômes et vers une amélioration de la formation des enseignants. Cette deuxième étape est d'ailleurs suivie, en 1942, d'une élévation des critères d'admission à l'École normale Macdonald où, dorénavant, seuls les détenteurs d'une $\mathrm{XI}^{\mathrm{e}}$ ou d'une

37 Nous utiliserons dorénavant, pour plus de commodité, les noms de brevets adoptés à la fin des années 1950 et conservés jusqu'à l'adoption du baccalauréat obligatoire, c'est-à-dire les brevets de classe I, II et III. De plus, il faut souligner qu'un brevet de spécialiste pour certaines matières, telles que les sciences ménagères, l'éducation physique ou le français, ainsi qu'un brevet pour enseigner au niveau de la maternelle, sont également accordés par le bureau central des examinateurs.

38 Comite protestant, The Regulations of the Protestant Committee of the Council of Education, 1931, article 13 (par la suite, art. 13); 1940, art. 118; 1951, art. 120. 
$\mathrm{XII}^{\mathrm{e}}$ année seront admis à suivre les cours de l'établissement. Ce relèvement des conditions d'admission, favorisé par l'instauration, en 1935, d'une XII ${ }^{e}$ année au programme des high schools, est mis en application alors que le personnel enseignant est touché par les départs massifs occasionnés par la Deuxième Guerre mondiale. En 1952, la création du baccalauréat en éducation et la possibilité pour les élèvesmaîtres, candidats au brevet de classe II, de bénéficier d'une période de formation de deux ans à l'école normale, marquent un nouveau tournant. D'une part, le nouveau baccalauréat offert par la Faculté des Arts et des Sciences de l'Université McGill permet une formation professionnelle continue sur quatre ans qui assure ainsi une meilleure préparation au travail d'enseignant. D'autre part, en plus d'offrir, mais sur une plus courte période, cette possibilité de formation professionnelle continue aux candidats du brevet de classe II, la mise en place du cours de deux ans à l'école normale témoigne aussi de la volonté de leur procurer une formation plus complète, à la fois générale et professionnelle, et surtout davantage tournée vers la préparation à l'enseignement ${ }^{39}$. L'abolition du brevet d'enseignement de classe III, en 1963, marque à son tour une étape importante. Réclamée depuis le début des années 1950 par l'Association provinciale des enseignants protestants, elle restreint à deux le nombre de diplômes accessibles et porte, par le fait même, à treize années le nombre minimum d'années de formation scolaire nécessaire à l'obtention d'un diplôme. Par la suite, l'adoption par le nouveau ministère de l'Éducation du règlement numéro quatre relatif au permis et au brevet d'enseignement, l'abolition des diplômes de classe II et I ainsi que le transfert complet de la formation des maîtres à l'université au cours de l'année scolaire 1970-1971 apparaissent comme l'aboutissement normal de cette série de transformations. Ils marquent l'étape ultime dans l'uniformisation et la centralisation de la formation des enseignants du secteur protestant et répondent aux recommandations du rapport Parent visant l'homogénéisation des structures du système scolaire québécois.

\section{Le niveau de certification du personnel enseignant}

Avant 1970, on observe un déplacement modéré du niveau de certification du personnel enseignant, du diplôme de troisième classe

39 Le directeur de l'École normale Macdonald note que, dès le milieu des années 1950, environ $25 \%$ des élèves-maîtres s'inscrivant aux cours menant au diplôme de classe II choisissent le programme de formation de deux ans; voir Département de l'Instruction publique, Mémoire présenté à la commission royale d'enquête sur les problèmes constitutionnels, juillet 1954, 55. 


\section{TABLEAU 4}

Nombre des enseignantes et enseignants des écoles protestantes publiques selon le sexe et le niveau de certification (1901-1965)

\begin{tabular}{|c|c|c|c|c|c|c|c|c|c|c|c|c|c|c|c|c|}
\hline \multirow[t]{2}{*}{ Année } & \multicolumn{4}{|c|}{ Classe III } & \multicolumn{4}{|c|}{ Classe II } & \multicolumn{4}{|c|}{ Classe I } & \multicolumn{4}{|c|}{ Autres" } \\
\hline & $H$ & $F$ & Ens & $\%$ & $H$ & $F$ & Ens & $\%$ & $H$ & $F$ & Ens & $\%$ & $H$ & $F$ & Ens & $\%$ \\
\hline $1900-1901$ & 7 & 702 & 709 & 55 & 23 & 436 & 459 & 36 & 58 & 64 & 122 & 9 & - & - & - & - \\
\hline $1910-1911$ & 14 & 625 & 639 & 47 & 47 & 530 & 577 & 42 & 68 & 75 & 143 & 11 & - & - & - & 0 \\
\hline $1920-1921$ & 6 & 582 & 588 & 33 & 28 & 969 & 997 & 55 & 105 & 107 & 212 & 12 & - & - & - & 0 \\
\hline $1930-1931$ & 8 & 591 & 599 & 26 & 61 & 1285 & 1346 & 57 & 170 & 231 & 401 & 17 & - & - & - & 0 \\
\hline $1934-1935$ & 17 & 588 & 605 & 24 & 93 & 1309 & 1402 & 55 & 238 & 296 & 534 & 21 & - & - & - & 0 \\
\hline $1940-1941$ & 14 & 414 & 428 & 17 & 118 & 1321 & 1439 & 58 & 278 & 334 & 612 & 25 & - & - & - & 0 \\
\hline $1944-1945$ & 14 & 577 & 591 & 25 & 116 & 947 & 1063 & 45 & 169 & 249 & 418 & 18 & 76 & 221 & 297 & 13 \\
\hline $1950-1951$ & 31 & 607 & 638 & 25 & 71 & 965 & 1036 & 41 & 382 & 276 & 658 & 26 & 86 & 118 & 204 & 8 \\
\hline $1954-1955$ & 39 & 842 & 881 & 29 & 86 & 975 & 1061 & 35 & 461 & 343 & 804 & 27 & 102 & 161 & 263 & 9 \\
\hline $1960-1961$ & 110 & 1168 & 1278 & 36 & 144 & 1121 & 1265 & 35 & 498 & 472 & 970 & 27 & 38 & 33 & 71 & 2 \\
\hline $1964-1965$ & 107 & 927 & 1034 & 24 & 289 & 1492 & 1781 & 41 & 730 & 700 & 1430 & 33 & 48 & 55 & 103 & 2 \\
\hline Moyenne & & & & 29 & & & & 45 & & & & 23 & & & & 3 \\
\hline
\end{tabular}

a Comprend, en 1950-1951, 1960-1961 et 1964-1965, les détenteurs de brevets de spécialistes; en 1954-1955, les détenteurs de certificats spéciaux.

Sources: RSDIP, 1900 à 1964; Statistiques de l'enseignement, 1944-1945, 1964-1965. 
vers celui de première classe (tableau 4). De 1901 à 1911, 51\% des enseignants brevetés détiennent, en moyenne, un diplôme de classe III, tandis qu'ils sont $54 \%$ à en détenir un de classe II au cours des vingt-cinq années suivantes. De 1945 à 1965, un équilibre relatif s'établit entre les détenteurs des diplômes respectifs, à la faveur d'une augmentation progressive du nombre de détenteurs du diplôme de classe I et de brevets de spécialistes. Cette tendance vers l'engagement d'enseignants de formation universitaire est encore plus marquée si l'on prend en compte les enseignants non légalement qualifiés détenant un diplôme universitaire, qui forment respectivement $34 \%$ et 49\% de ces enseignants en 1960-1961 et $1964-1965^{40}$. L'enquête de H. J. Martin sur les enseignants du niveau élémentaire confirme d'ailleurs cette tendance. Elle souligne que les enseignants qui détiennent un diplôme de classe III, en 1963-1964, soit 27\% des 2217 répondants au questionnaire, sont en place depuis plusieurs années, voire pour un grand nombre d'entre eux depuis plus de vingt ans, et que $10 \%$ de ceux qui ont déclaré avoir commencé leur carrière avec un tel diplôme possèdent au moment de l'enquête un diplôme de classe I ou de classe $\mathrm{II}^{41}$.

L'augmentation du nombre de diplômés des classes II et I conforte l'hypothèse voulant que l'idée d'une formation universitaire pour les enseignants de tous les niveaux scolaires jouisse d'une popularité grandissante à partir des années 1950, tant chez les enseignants que chez les commissaires d'écoles ${ }^{42}$. L'abandon du diplôme de troisième classe, en 1963, n'apparaît plus alors que comme une conclusion logique de la dévalorisation progressive de ce diplôme et de la recherche constante, depuis les années 1940, d'un accroissement de la durée de la formation générale et professionnelle de l'enseignant. Cette tendance à l'augmentation progressive du nombre d'années de formation générale et professionnelle des enseignants est bien réelle. Tandis que jusqu'en 1909, neuf années d'études sont suffisantes pour l'obtention du brevet minimum ${ }^{43}$, il en faut treize en $1963^{44}$ et, avec l'instauration, au cours des années 1960, du baccalauréat obligatoire et l'ajout des deux années de formation préuniversitaire du CÉGEP, le

40 Calcul fait à partir de RSDIP (1960-1961): 258 et Statistiques de l'enseignement, 1964-1965, 254.

41 H. J. Martin, «Survey Summary Analyses Teachers' Qualification», Teachers' Magazine (nov. 1968): 38.

42 Munroe, loc. cit. (1957), 11; «Rapport du directeur de l'Institut pédagogique», dans RSDIP (1960-1961): 51.

43 Comité protestant, The Regulations of the Protestant Committee of the Council of Education (1909), article 26a.

44 F. Crook, op. cit. (1962), 5 et 8. 
nombre d'années de scolarité minimum requis pour accéder à la profession enseignante est désormais de seize ${ }^{45}$. Ces transformations se reflètent d'ailleurs sur le nombre moyen d'années de scolarité des enseignants. Tandis qu'il est de 13,6 au milieu des années soixante, il atteint vingt ans plus tard le seuil de $16,7^{46}$.

Plusieurs facteurs expliquent l'élévation progressive du niveau de certification. Certains ont trait aux changements touchant directement les conditions d'émission des diplômes, d'autres aux pressions exercées par la direction de l'enseignement protestant pour élever le niveau de formation de son personnel enseignant. D'autres facteurs encore relèvent davantage des transformations du système scolaire luimême. La diversification croissante du programme d'études des écoles secondaires, qui favorise la présence de plus en plus grande de spécialistes dans les écoles, ainsi que l'augmentation de leur clientèle sont autant d'éléments ayant pu encourager ce mouvement.

Les enseignants de sexe masculin - regroupés, comme nous l'avons vu, principalement dans les écoles secondaires - reçoivent en moyenne, jusqu'au milieu des années soixante, une formation plus longue que leurs collègues de sexe féminin. Entre 1901 et 1965 , de $45 \%$ à $76 \%$ d'entre eux détiennent un diplôme de première classe alors que seulement de $5 \%$ à $22 \%$ des enseignantes détiennent ce même diplôme (tableau 5). La majorité de ces dernières opte plutôt pour des diplômes qui ne nécessitent qu'une ou deux années de formation, comme les diplômes de deuxième et troisième classe. Cette tendance, chez les hommes, à une plus longue formation générale et professionnelle entraîne des conséquences importantes, entre autres, sur les conditions de travail des enseignants, car le niveau de certification se révèle être un des éléments de base des échelles salariales graduellement adoptées par les différentes commissions scolaires, puis par le ministère de l'Éducation, au cours de la période étudiée. Elle affecte également, pour certaines personnes, l'organisation même de la carrière, puisque c'est généralement parmi les détenteurs de diplômes de première classe qu'est recruté le personnel de direction et de supervision pédagogique. En 1961, par exemple, ils constituent $85 \%$ du personnel non enseignant, chez les hommes, et $50 \%$, chez les femmes. À l'inverse, seulement $1 \%$ du personnel non enseignant masculin et $8 \%$ du côté féminin détiennent un diplôme de troisième classe.

45 Burgess, op. cit. (1977), 148.

46 Calculs effectués à partir des Statistiques de l'enseignement, 1964-1965, 260 et de MEQ, Personnel de la commission scolaire (fichier PERCOS), 1985. 
TABLEAU 5

Répartition en pourcentage du personnel enseignant des écoles protestantes publiques selon le niveau de certification

(1901-1965)

\begin{tabular}{lrccccccc}
\hline Année & \multicolumn{3}{c}{ Homme } & \multicolumn{5}{c}{ Femme } \\
\hline & \multicolumn{1}{l}{ III } & $I I$ & $I$ & autres & III & $I I$ & $I$ & autres \\
\cline { 2 - 9 } $1900-1901$ & 8 & 26 & 66 & - & 58 & 36 & 5 & - \\
$1910-1911$ & 11 & 36 & 53 & - & 51 & 43 & 6 & - \\
$1920-1921$ & 4 & 20 & 76 & - & 35 & 58 & 6 & - \\
$1930-1931$ & 3 & 26 & 71 & - & 28 & 61 & 11 & - \\
$1934-1935$ & 5 & 27 & 68 & - & 27 & 60 & 13 & - \\
$1940-1941$ & 3 & 29 & 68 & - & 20 & 64 & 16 & - \\
$1944-1945$ & 4 & 31 & 45 & 20 & 29 & 47 & 12 & 11 \\
$1950-1951$ & 5 & 12 & 67 & 15 & 31 & 49 & 14 & 6 \\
$1954-1955$ & 6 & 13 & 67 & 15 & 36 & 42 & 15 & 7 \\
$1960-1961$ & 14 & 18 & 63 & 5 & 42 & 40 & 17 & 1 \\
$1964-1965$ & 9 & 25 & 62 & 4 & 29 & 47 & 22 & 2 \\
\hline
\end{tabular}

Sources: Calculs fait à partir du tableau 4.

\section{L'expérience dans l'enseignement}

Les statistiques sur le nombre d'années d'expérience des enseignants montrent que le système scolaire protestant n'a connu que deux génération $\mathrm{s}^{47} \mathrm{~d}$ 'enseignants au cours du $\mathrm{XX}^{\mathrm{e}}$ siècle: une première, qui va de la fin du XIX ${ }^{\mathrm{e}}$ siècle jusque vers la fin des années 1940 et le début des années 1950; une seconde, émergeant au cours des années 1950-1960, à la faveur du développement que connaît le système scolaire québécois dans son ensemble, et qui se prolonge jusqu'à l'orée des années 1990 (graphique 2). Si l'on compare, par exemple, la proportion des enseignants ayant quinze années et plus d'expérience, en 1915, avec celle de 1945, on constate une augmentation de $24 \%$, tandis qu'une augmentation de 34\% est enregistrée entre 1961 et 1989.

Même si elle n'explique pas tout, la mise à jour de l'existence de ces deux générations permet d'apporter un éclairage nouveau sur la pénurie d'enseignants qui frappe le système scolaire protestant, surtout après les années 1940, ainsi que sur le succès de la réforme scolaire des années 1960. Le vieillissement de la population enseignante, au cours des années 1940, jumelé à une diminution de la proportion de

47 «Génération» doit être entendu ici au sens large du terme et non au sens démographique. 
GRAPHIQUE 2

Répartition en pourcentage du personnel enseignant des écoles protestantes publiques selon les années d'expériences, 1915-1989.

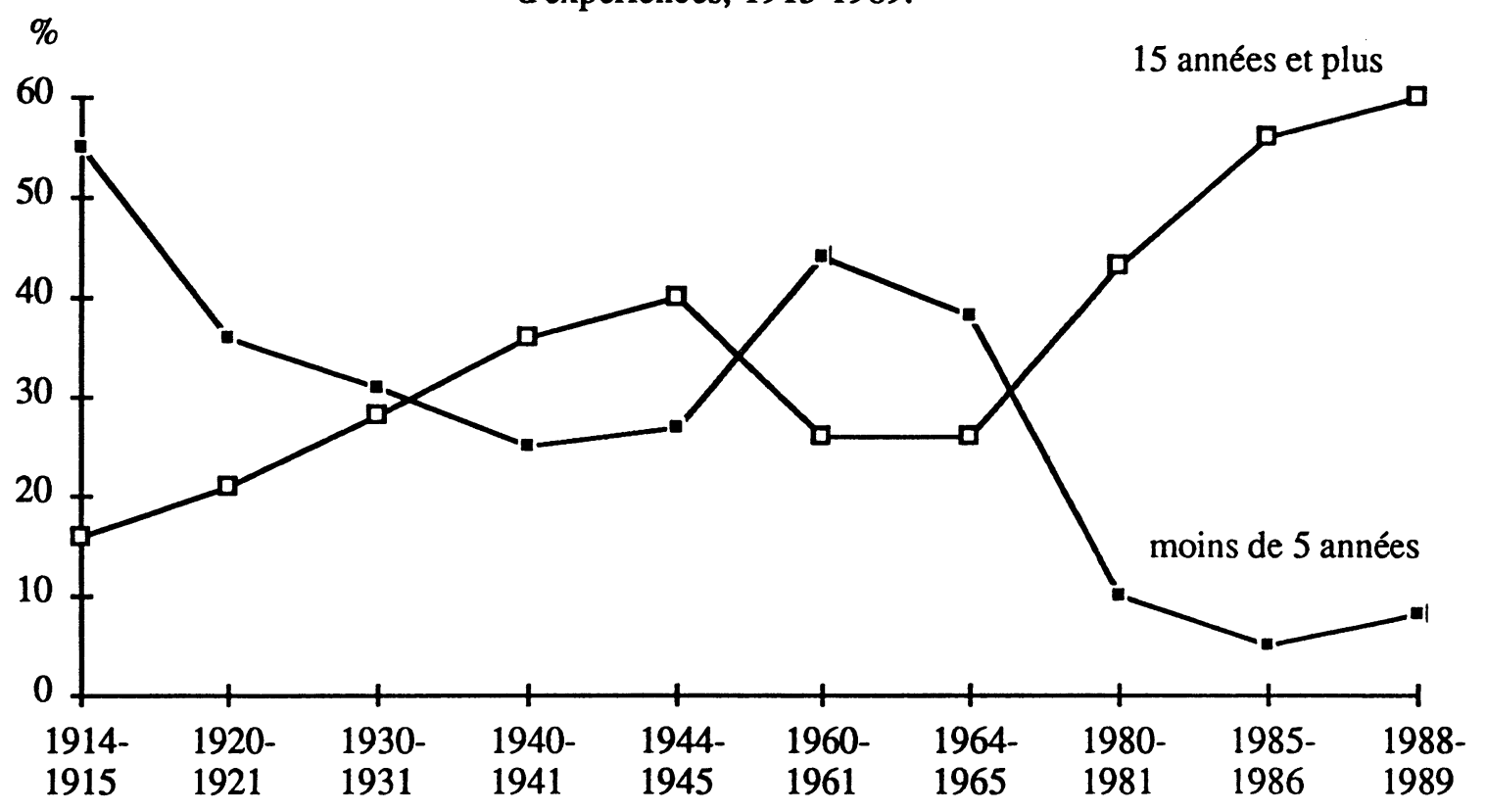

Sources: Statistiques de l'enseignement, 1921-1922 à 1944-1945, 1964-1965; RSDIP, 1960-61; Ministère de l'Éducation du Québec, Personnel de la commission scolaire (fichier PERCOS), 1980, 1985, 1989. 
jeunes recrues, peut en effet expliquer, en partie, l'essoufflement du recrutement signalé à partir de cette époque. On peut constater, en revanche, que la réforme scolaire des années 1960 , contrairement à celle des années 1930, a pu bénéficier d'un corps enseignant renouvelé, moins expérimenté et probablement plus sensibilisé aux nouvelles méthodes pédagogiques.

Comparées entre elles, les données sur les années d'expérience des enseignants des deux sexes suivent à peu de choses près le même mouvement (tableau 6). Toutefois, les hommes ont tendance, avant le milieu des années 1940, à rester plus longtemps dans la profession que les femmes. Cette tendance se renverse cependant dans le deuxième tiers du siècle, pour revenir à la situation initiale au cours des années 1980. Il apparaît évident que les motifs pour demeurer dans l'enseignement peuvent être variés. La durée du maintien dans la profession peut prendre des significations différentes selon la position qu'occupent les acteurs dans les rapports sociaux et la période où ils occupent cette position. Les enseignants d'aujourd'hui, par exemple, ont peu à voir avec ceux du début du siècle: meilleurs salaires, meilleure sécurité d'emploi, meilleures conditions de travail et de retraite, peu de différence avec les salariés des autres secteurs ayant les mêmes qualifications qu'eux, etc.

L'accessibilité des postes administratifs tend également à varier, au cours de la période. Cependant, il est possible que l'attrait de la carrière administrative ait incité certains des enseignants de sexe masculin à rester plus longtemps en place, sachant qu'un autre échelon peut être gravi. Cette situation conforte d'ailleurs l'opinion émise par certains administrateurs scolaires, à partir des années cinquante, selon laquelle plus la période de formation des enseignants est longue, plus ceux-ci restent longtemps dans la profession ${ }^{48}$. Le retrait fréquent des enseignantes, principalement afin d'élever leur famille, peut également constituer un élément d'explication de l'écart entre les années d'expérience des hommes et des femmes. Près de $16 \%$ d'entre elles s'absentent de la profession pendant plus de dix ans, selon l'enquête de F. Crook menée au début des années soixante ${ }^{49}$.

D'ailleurs, dès le milieu des années 1950, les femmes mariées forment plus de la moitié du personnel enseignant féminin du secteur protestant $^{50}$. Leur présence est massive, comparée à celle des enseignantes mariées du secteur catholique. L'écart entre le nombre

48 Munroe, loc. cit. (1957), 10-11.

49 Ce taux est inférieur à $2 \%$ chez les hommes; Crook, op. cit. (1962), 36-37.

50 RSDIP (1959-1960): 43. 


\section{TABLEAU 6}

Répartition en pourcentage du personnel enseignant laïque des écoles publiques catholiques et protestantes selon les années d'expérience (1915-1989)

\begin{tabular}{|c|c|c|c|c|c|c|c|c|c|c|c|c|c|c|c|c|}
\hline \multirow[t]{3}{*}{ Année } & \multicolumn{4}{|c|}{$1-4$ ans } & \multicolumn{4}{|c|}{$5-9$ ans } & \multicolumn{4}{|c|}{$10-14$ ans } & \multicolumn{4}{|c|}{15 ans et plus } \\
\hline & \multicolumn{2}{|c|}{ Hommes } & \multicolumn{2}{|c|}{ Femmes } & \multicolumn{2}{|c|}{ Hommes } & \multicolumn{2}{|c|}{ Femmes } & \multicolumn{2}{|c|}{ Hommes } & \multicolumn{2}{|c|}{ Femmes } & \multicolumn{2}{|c|}{ Hommes } & \multicolumn{2}{|c|}{ Femmes } \\
\hline & $C$ & $P$ & $C$ & $P$ & $C$ & $P$ & $C$ & $P$ & $C$ & $P$ & $C$ & $P$ & $C$ & $P$ & $C$ & $P$ \\
\hline $1914-1915^{a}$ & 34 & 51 & 61 & 56 & 25 & 10 & 24 & 21 & 9 & 9 & 7 & 9 & 32 & 30 & 8 & 14 \\
\hline $1920-1921^{\star}$ & 29 & 23 & 65 & 38 & 28 & 28 & 21 & 29 & 17 & 19 & 6 & 13 & 26 & 30 & 7 & 20 \\
\hline $1930-1931^{*}$ & 21 & 23 & 56 & 32 & 24 & 29 & 26 & 26 & 22 & 17 & 10 & 15 & 34 & 31 & 8 & 27 \\
\hline $1940-1941^{a}$ & 14 & 25 & 44 & 26 & 21 & 23 & 29 & 22 & 21 & 18 & 13 & 16 & 44 & 34 & 14 & 36 \\
\hline $1944-1945$ & 12 & 23 & 47 & 28 & 20 & 14 & 25 & 20 & 20 & 19 & 12 & 13 & 48 & 44 & 14 & 39 \\
\hline $1950-1951$ & - & - & - & - & - & - & - & - & - & - & - & - & - & - & - & - \\
\hline $1954-1955$ & - & - & - & - & - & - & - & - & - & - & - & - & - & - & - & - \\
\hline $1960-1961$ & 38 & 47 & 46 & 42 & 24 & 22 & 25 & 18 & 13 & 13 & 14 & 12 & 25 & 18 & 15 & 29 \\
\hline $1964-1965$ & 48 & 40 & 42 & 37 & 26 & 25 & 29 & 23 & 11 & 15 & 13 & 11 & 15 & 20 & 16 & 29 \\
\hline $1970-1971$ & - & - & - & - & - & - & - & - & - & - & - & - & - & - & - & - \\
\hline $1974-1975$ & - & - & - & - & - & - & - & - & - & - & - & - & - & - & - & - \\
\hline $1980-1981$ & 4 & 7 & 6 & 10 & 15 & 21 & 12 & 26 & 32 & 21 & 24 & 23 & 49 & 51 & 58 & 39 \\
\hline $1985-1986$ & 3 & 3 & 5 & 5 & 8 & 10 & 10 & 18 & 17 & 22 & 15 & 24 & 72 & 65 & 70 & 51 \\
\hline $1988-1989$ & 5 & 5 & 7 & 8 & 5 & 6 & 9 & 14 & 11 & 16 & 13 & 22 & 78 & 75 & 71 & 54 \\
\hline
\end{tabular}

a Ne comprend que le personnel breveté.

Source: Statistiques de l'enseignement, 1921-1922 à 1944-1945, 1964-1965; RSDIP, 1960-1961; ministère de l'Éducation du Québec, Personnel de la commission scolaire (fichier PERCOS), 1980, 1985, 1989. 
d'années d'expérience des enseignantes protestantes et celui des enseignantes laïques du secteur catholique est flagrant et significatif (tableau 6). Tandis que les femmes catholiques, avant les années 1960, ne restent dans la profession que six ans en moyenne, soit jusqu'au moment de leur mariage, les protestantes semblent bénéficier d'une plus grande accessibilité au marché du travail enseignant. La proportion d'enseignantes protestantes possédant quinze années et plus d'expérience est, jusqu'à cette période, presque continuellement le double de celle des catholiques, tandis qu'avant les années 1950, la proportion de ces dernières parmi les enseignantes ayant moins de cinq années d'expérience est toujours supérieure en moyenne à celle des protestantes. Cette tendance semble s'inverser, après les années 1960 , et le vieillissement de la population enseignante féminine du secteur catholique tend à être plus important que chez les protestants ${ }^{51}$.

\section{CONDITIONS SALARIALES}

La question des conditions salariales du personnel enseignant est étroitement liée à celles de son recrutement et de sa qualification. Elle constitue, jusqu'au début des années 1950, la pierre angulaire autant du discours du comité protestant que de celui des inspecteurs d'écoles et de l'Association provinciale des enseignants protestants. C'est sur ces conditions salariales que semble, en fait, reposer toute l'amélioration de la condition enseignante et, par elle, celle du système scolaire lui-même.

\section{Mécanismes de négociation}

L'histoire des mécanismes de négociation salariale dans le secteur protestant peut se découper en deux grandes périodes: celle qui précède le début des réformes scolaires des années 1960, et celle qui la suit. Au cours de la première période, les mécanismes reposent exclusivement sur une base locale et régionale: les commissions scolaires voient à l'embauche et à la rémunération des enseignants.

51 Il faut cependant noter que jusque dans les années trente, et peut-être même au-delà, les statistiques officielles n'ont jamais pris en compte les années d'expérience acquises à l'extérieur de la province. Pourtant, comme le montre l'enquête de F. Crook, cette part d'expérience est fort appréciable puisqu'un grand nombre d'enseignants non québécois sont engagés par les commissions scolaires protestantes du Québec; ibid., 31. De plus, les statistiques officielles d'avant 1945 ne prenant en compte que les enseignants brevetés, cette politique a tendance à diminuer la proportion d'enseignants peu expérimentés. À titre d'exemple, en 1944$1945,89 \%$ des enseignants non brevetés comptent moins de cinq années d'expérience, tandis que ce taux, chez les détenteurs de brevet, n'atteint que 22\%; Statistiques de l'enseignement, 1944$1945,209,213,219$ et 223. 
Les contrats sont généralement renouvelables d'année en année et négociés sur une base individuelle tandis que les enseignants ne sont payés que pour les mois de travail, dont le nombre peut varier selon les régions. La sécurité d'emploi, surtout en milieu rural, est pratiquement inexistante. Certaines commissions scolaires rurales pratiquent ce qu'on a appelé, en langage populaire, une politique de «hiring and firing», c'est-à-dire une politique de congédiement annuel systématique. Cette politique oblige les enseignants à concourir chaque année avec les nouveaux venus pour obtenir un poste et permet aussi aux commissions scolaires de ne réengager que le personnel qu'elles désirent effectivement garder. De tels congédiements massifs sont facilités par l'absence d'obligation, pour les commissaires, d'en fournir les raisons au département de l'Instruction publique et aux enseignants eux-mêmes ${ }^{52}$.

$\mathrm{Au}$ cours de cette période, il n'existe aucune échelle salariale commune pour l'ensemble des commissions scolaires de la province. De l'avis de certains observateurs, il serait d'ailleurs impossible, voire non souhaitable d'en établir une, compte tenu de la grande diversité qui caractérise, entre autres, le milieu rural. Chaque commission scolaire rurale, selon le comité protestant, doit pouvoir adopter une politique salariale conforme à ses ressources. De plus, la direction de l'enseignement protestant craint que l'adoption d'une telle échelle salariale protège abusivement les enseignants médiocres.

Cependant, à partir de 1952, une échelle salariale globale, proposée par l'Association des enseignants, est finalement adoptée par un grand nombre de commissions scolaires rurales pour servir de guide dans leurs négociations salariales. Dès 1950-1951, 43\% d'entre elles avaient déjà offert cette échelle à leurs enseignants ${ }^{53}$. Son implantation marque l'amorce d'une uniformisation des conditions salariales. Elle permet aux salaires des enseignants œuvrant en milieu rural de s'uniformiser et de se rapprocher graduellement de ceux de leurs confrères et consœurs du milieu urbain: d'abord, en proposant des augmentations plus élevées aux enseignants du milieu rural, ensuite et surtout, en faisant reposer l'augmentation de leurs salaires sur les mêmes paramètres que ceux adoptés depuis plusieurs années par la Montreal Protestant Central School Board (MPCSB).

Dans cette mécanique salariale, le comité protestant joue un rôle d'intermédiaire ${ }^{54}$. Il aide les enseignants, représentés par la PAPT, à

52 W. A. F. Hepburn, op. cit. (1938), 219.

53 Talbot, op. cit. (1964), 72.

54 Mair, op. cit. (1981), 73. 
piloter leurs revendications et voit à exercer régulièrement des pressions sur les autorités scolaires locales et sur le gouvernement provincial. Aux commissions scolaires qui lui disent ne pas disposer d'assez de revenus pour accéder aux demandes des enseignants, et qu'il menace parfois de coercition ${ }^{55}$, il répond par des demandes réitérées de subventions auprès du gouvernement et du département de l'Instruction publique. Les fonds nécessaires au paiement des enseignants sont constitués, en fait, à la fois des taxes scolaires locales et des subventions gouvernementales.

D'importantes transformations affectent les mécanismes de négociation après l'adoption de la grande charte de l'Éducation (19601961). Le droit de grève, qui leur était jusqu'alors interdit, est accordé aux enseignants en 1965 tandis que, depuis 1963, ils peuvent demander des explications sur les raisons de leur congédiement et même faire appel à un tribunal d'arbitrage, s'ils ont plus de trois ans de service dans la même commission scolaire ${ }^{56}$. Les enseignants protestants ne se sont toutefois jamais prévalus de ces nouveaux amendements au Code du travail. Ils ont plutôt continué à négocier à l'amiable avec les commissions scolaires locales. Pour eux, la grande rupture s'effectue en 1967 avec l'adoption de la loi 25. Mettant fin à une série de grèves et de ralentissements de travail, qui déséquilibraient l'ensemble du système scolaire québécois depuis le début des années 1960, cette loi modifie de façon substantielle les mécanismes traditionnels de négociation entre l'enseignant et son employeur. Elle impose un déplacement géographique de la négociation ainsi qu'une transformation des structures de pouvoir des différents intervenants. La négociation locale ou régionale cède le pas à la négociation provinciale. Les commissions scolaires voient leur dépendance face au gouvernement s'agrandir, tandis que les associations locales d'enseignants doivent s'en remettre à l'association provinciale que forme la PAPT. Tout est centralisé autour du gouvernement et des représentants provinciaux des commissions scolaires et des enseignants. Les ententes sur une base individuelle disparaissent au profit d'une convention collective.

55 En 1912, par exemple, on applique une réduction d'un cinquième de point dans l'évaluation du comité protestant pour chaque tranche de $5 \$$ nécessaires à l'augmentation du salaire des directeurs et des enseignants de high schools jusqu'au seuil minimum convenu. Ce qui signifiait une diminution comparable des subventions gouvernementales. Voir, W. P. Percival, Across the Years. A Century of Education in the Province of Quebec (Montréal, Gazette Printing Company ltd., 1946), 110.

56 Commission royale d'enquête sur l'enseignement dans la province de Québec, Rapport, 5 (1966): 217. 
La loi 25, jumelée à l'adoption en 1966 et 1968 des règlements numéro quatre et cinq sur la certification des enseignants et leur classification pour fins salariales, apporte également des modifications sensibles à l'identité et au statut social de l'enseignant protestant. Par l'établissement d'une échelle salariale, d'une certification et d'une classification communes à l'ensemble du personnel enseignant de la province, toutes confessions religieuses confondues, il uniformise les modalités d'accès à la profession, lieu d'identification fort qui fut et est toujours un enjeu de pouvoir important. Les autorités scolaires locales, régionales et provinciales, les associations d'enseignants et les établissements de formation ont toujours voulu définir à leur façon ces modalités. Définir les modalités d'accès à la profession, c'est en définir le caractère identitaire, la personnalité et l'avenir. Avec l'uniformisation de ces modalités, les spécificités professionnelles et salariales du corps enseignant des deux secteurs confessionnels tendent à s'effacer. De plus, longtemps préoccupé par le besoin de se définir une place dans la société et d'obtenir une reconnaissance du public, sans vraiment réellement y parvenir, le corps enseignant, à la suite de l'intervention gouvernementale à titre d'employeur, est brusquement assimilé au groupe en pleine expansion que constituent les fonctionnaires de l'État ${ }^{57}$.

\section{Variations statistiques des conditions salariales}

L'analyse statistique du salaire moyen des enseignants du secteur protestant révèle l'existence de trois phases (tableau 7). La première s'échelonne du début du siècle jusqu'au début des années 1920. Elle est marquée par un taux de croissance progressif, généralisé à l'ensemble du personnel enseignant, mais particulièrement remarquable chez les femmes où il varie entre $182 \%$ et $283 \%$. Cette hausse du salaire moyen est attribuable, entre autres, à l'intervention favorable $\mathrm{du}$ gouvernement de Lomer Gouin pour permettre aux commissions scolaires rurales de se rapprocher le plus possible du salaire minimum de 60\$ par mois suggéré, en 1920, par le comité protestant à la requête de la PAPT ${ }^{58}$. Ce salaire minimum, qui n'a jamais été obligatoire, peut à peine concurrencer les salaires offerts par certaines compagnies privées à leurs secrétaires et commis. Par exemple, en 1918, une sténographe de la Canadian Rubber Company est payée entre $50 \$$ et $90 \$$

57 R. E. Lavery, "Changes in the Teaching Profession», McGill Journal of Education, 7,2 (aut. 1972): 170 .

58 A. D. Talbot, PAPT: The First Century: A History of the Provincial Association of Protestant Teachers of Quebec (Gardenvale (Québec), Harpell's Press, 1964), 50; «Rapport de l'inspecteur général des écoles protestantes», RSDIP (1920-1921): 257. 


\section{TABLEAU 7}

Salaire moyen (\$) du personnel enseignant des écoles protestantes publiques et taux d'augmentation périodique (Aug.) selon le niveau d'enseignement, le sexe et le milieu social (1901-1989)

\begin{tabular}{|c|c|c|c|c|c|c|c|c|}
\hline \multirow[t]{2}{*}{ Année } & \multicolumn{4}{|c|}{ Urbain } & \multicolumn{4}{|c|}{ Rural } \\
\hline & $H$ & Aug & $F$ & Aug & $H$ & Aug & $F$ & Aug \\
\hline & & $\%$ & & $\%$ & & $\%$ & & $\%$ \\
\hline \multicolumn{9}{|c|}{ Élémentaire } \\
\hline $1900-1901^{\circ}$ & 1149 & 0 & 367 & 0 & 205 & 0 & 149 & 0 \\
\hline $1910-1911^{a}$ & 1410 & 23 & 511 & 39 & 600 & 193 & 233 & 56 \\
\hline $1920-1921^{a}$ & 2648 & 88 & 1205 & 136 & 2233 & 272 & 570 & 145 \\
\hline $1930-1931^{\mathrm{a}}$ & 2761 & 4 & 1265 & 5 & 605 & -73 & 553 & -3 \\
\hline $1934-1935^{\mathrm{a}}$ & 2319 & -16 & 1141 & -10 & 382 & -37 & 390 & -29 \\
\hline $1940-1941^{2}$ & 2531 & 9 & 1247 & 9 & 605 & 58 & 502 & 29 \\
\hline $1950-1951$ & - & - & - & - & - & - & - & - \\
\hline $1960-1961$ & 4263 & 68 & 3774 & 203 & 2589 & 328 & 3098 & 517 \\
\hline 1964-1965 & 4885 & 15 & 4586 & 22 & 3518 & 36 & 3877 & 25 \\
\hline $1970-1971$ & - & - & - & - & - & - & - & - \\
\hline $1980-1981^{b}$ & 23460 & 380 & 20979 & 357 & 23460 & 567 & 20979 & 441 \\
\hline $1985-1986^{b}$ & 31569 & 35 & 27288 & 30 & 31569 & 35 & 27288 & 30 \\
\hline $1988-1989^{b}$ & 33023 & 5 & 30741 & 13 & 33023 & 5 & 30741 & 13 \\
\hline \multicolumn{9}{|c|}{ Secondaire } \\
\hline $1900-1901^{\mathrm{a}}$ & 1075 & 0 & 410 & 0 & 628 & 0 & 264 & 0 \\
\hline $1910-1911^{\mathrm{a}}$ & 1358 & 26 & 479 & 17 & 968 & 54 & 374 & 42 \\
\hline $1920-1921^{\mathrm{a}}$ & 2249 & 66 & 1156 & 141 & 1679 & 73 & 770 & 106 \\
\hline $1930-1931^{\text {a }}$ & 2744 & 22 & 1352 & 17 & 1614 & -4 & 803 & 4 \\
\hline $1934-1935^{a}$ & 2331 & -15 & 1141 & -16 & 1056 & -35 & 390 & -51 \\
\hline $1940-1941^{\mathrm{a}}$ & 2667 & 14 & 1399 & 23 & 1266 & 20 & 707 & 81 \\
\hline $1950-1951$ & - & - & - & - & - & - & - & - \\
\hline 1960-1961 & 6133 & 130 & 5330 & 281 & 5251 & 315 & 4061 & 474 \\
\hline 1964-1965 & 7002 & 14 & 6162 & 16 & 5716 & 9 & 4490 & 11 \\
\hline $1970-1971$ & & & & - & - & - & - & - \\
\hline $1980-1981^{b}$ & 26041 & 272 & 23272 & 278 & 26041 & 356 & 23272 & 418 \\
\hline $1985-1986^{b}$ & 33795 & 30 & 31278 & 34 & 33795 & 30 & 31278 & 34 \\
\hline $1988-1989^{b}$ & 36586 & 8 & 32883 & 5 & 36586 & 8 & 32883 & 5 \\
\hline
\end{tabular}

a Statistiques du personnel enseignant breveté des écoles publiques et privées.

b Les statistiques ne distinguent pas le milieu social pour cette période.

Sources: RSDIP 1900 à 1961; Statistiques de l'enseignement, 1964-1965, 1970-1971; ministère de l'Éducation du Québec, Personnel de la commission scolaire (fichier PERCOS), 1980. 1985, 1989. 


\section{TABLEAU 8}

Salaire moyen (\$) du personnel enseignant laïque des écoles catholiques $(\mathrm{C})$ et protestantes $(\mathrm{P})$ publiques selon le sexe (1921-1989)

\begin{tabular}{|c|c|c|c|c|c|c|c|c|}
\hline \multirow[t]{2}{*}{ Année } & \multicolumn{4}{|c|}{ Hommes } & \multicolumn{4}{|c|}{ Femmes } \\
\hline & $C$ & Indice & $P$ & Indice & $C$ & Indice & $P$ & Indice \\
\hline $1920-1921^{\mathrm{a}}$ & 1280 & 100 & 2300 & 100 & 308 & 100 & 1013 & 100 \\
\hline $1925-1926^{\mathrm{a}}$ & 1434 & 112 & 2279 & 99 & 367 & 119 & 1047 & 103 \\
\hline $1930-1931^{a}$ & 1647 & 129 & 2596 & 113 & 402 & 131 & 1127 & 111 \\
\hline $1934-1935^{a}$ & 1459 & 114 & 2034 & 88 & 315 & 102 & 980 & 97 \\
\hline $1940-1941^{\mathrm{a}}$ & 1787 & 140 & 2382 & 104 & 419 & 136 & 1111 & 110 \\
\hline 1951-1952 & 2929 & 229 & - & - & 975 & 317 & 1695 & - \\
\hline 1954-1955 & 3443 & 269 & - & - & 1473 & 478 & - & - \\
\hline $1960-1961^{b}$ & 4989 & 390 & 6154 & 268 & 2555 & 830 & 4174 & 412 \\
\hline 1964-1965 & 6465 & 505 & 7123 & 310 & 4032 & 1309 & 5104 & 504 \\
\hline 1970-1971 & - & - & - & - & - & - & - & - \\
\hline 1974-1975 & - & - & - & - & - & - & - & - \\
\hline 1980-1981 & 25740 & 20112 & 26379 & 1147 & 22546 & 73202 & 22759 & 2247 \\
\hline 1985-1986 & 33575 & 26233 & 34199 & 1487 & 28737 & 93302 & 29272 & 2890 \\
\hline 1988-1989 & 37072 & 28963 & 37450 & 1628 & 31557 & 102463 & 32095 & 3168 \\
\hline
\end{tabular}

a Comprend le salaire moyen du personnel enseignant breveté des écoles privées.

b Comprend le salaire moyen des directeurs.

Sources: RSDIP 1960 à 1961; Statistiques de l'enseignement, 1920-1921 à 19641965; ministère de l'Éducation du Québec, Personnel de la commission scolaire (fichier PERCOS), 1980. 1985, 1989.

par mois tandis que la même personne, employée par la Canadian Pacific Railway, reçoit pour une période équivalente entre $62,50 \$$ et $150 \$$. De plus, plusieurs enseignants des provinces voisines reçoivent déjà, à la fin des années 1910, un salaire minimum de près de $70 \$$ par mois $^{59}$.

La victoire n'est que de courte durée pour les enseignants. La période qui suit cette hausse de 1920-1921 en est une de faible croissance. Entre 1921 et 1941, l'augmentation du salaire moyen des enseignantes est de $10 \%$, tandis que celle des enseignants n'atteint que $4 \%$ (tableau 8). La crise économique qui sévit à l'échelle mondiale et les problèmes financiers qui en résultent pour les commissions scolaires sont grandement responsables de cet état de fait. À Montréal, par exemple, aucune nouvelle échelle salariale n'est adoptée par la 
Montreal Protestant Central School Board avant 1942. Les augmentations prévues par les dernières échelles salariales en 1929, pour les enseignants des high schools, et en 1921, pour ceux des écoles élémentaires, sont interrompues après 1933 tandis qu'une diminution des salaires de $10 \%$ est décrétée l'année suivante ${ }^{60}$. Aux dires de W. Hepburn, dans son rapport remis au comité protestant en 1938, la situation est particulièrement critique chez les enseignants du niveau élémentaire de cette région, surtout parmi les femmes et les jeunes hommes mariés ${ }^{61}$. Le salaire moyen des enseignants de l'ensemble de la province, au plus fort de la crise, soit de 1931 à 1935, a diminué en moyenne de $22 \%$ chez les hommes et de $13 \%$ chez les femmes (tableau 8). Toutefois, c'est en milieu rural que les plus forts contrecoups de cette crise économique se font sentir. Avec la diminution de la population protestante et les difficultés qui frappent les exploitations agricoles et maritimes, la perception des taxes nécessaires au paiement des enseignants est perturbée ${ }^{62}$. Tandis que dans la première moitié des années 1930, le salaire moyen en milieu urbain subit une baisse d'environ $15 \%$, celui octroyé en milieu rural est affecté, de son côté, d'une diminution de plus de $38 \%$, en moyenne (tableau 7).

C'est encore une fois l'intervention du gouvernement provincial qui permet aux salaires de reprendre leur croissance par la suite. À l'aide de subventions accordées aux commissions scolaires rurales et urbaines, ainsi qu'avec la résorption de certaines dettes affectant, entre autres, la MPCSB, ils amorcent, dès le milieu des années 1940, un mouvement ascendant particulièrement rapide, surtout entre 1960 et 1980. Le tableau 8 témoigne, en effet, d'une progression de $158 \%$ chez les hommes et de $276 \%$ chez les femmes, entre 1941 et 1961 , et respectivement de $329 \%$ et de $445 \%$, entre 1961 et 1981 .

Outre la conjoncture sociale et économique favorable à l'amélioration du salaire du personnel enseignant, trois événements importants doivent être considérés. D'abord, l'adoption en 1952 de la première échelle salariale commune pour la majorité des commissions scolaires protestantes rurales, puis l'imposition en 1965-1966 d'un nouveau système de péréquation à la grandeur de la province ainsi que l'adoption de la loi 25 en 1967. Ces trois événements ont, à leur façon, modifié les règles de calcul de la rétribution du travail enseignant. Dans le premier cas, il s'est agi de remplacer l'arbitraire et les particularismes par la généralisation d'une méthode de calcul de la

60 Hepburn, op. cit., 222-223.

61 Ibid., 223.

62 Rapport de l'inspecteur général des écoles protestantes, RSDIP (1930-1931): 144. 
condition salariale reposant sur des paramètres précis qui permettent un ajustement rationnel des salaires. Dans le deuxième cas, c'est toute l'idéologie de l'égalité des chances d'accès à l'éducation et de l'équité salariale qui s'impose en mettant sur un même pied d'égalité, sur le plan budgétaire, les commissions scolaires catholiques et protestantes, et en imposant un plafond aux dépenses salariales de chacune. Dans le troisième cas, c'est tout le cadre référentiel des salaires qui bascule. Jusque-là comparée aux salaires des employés de bureau des compagnies privées et à celui des salariés de l'industrie, la rémunération du personnel enseignant est mise en regard avec l'échelle salariale des fonctionnaires de l'État, auxquels sont dorénavant associés les enseignants.

D'autres éléments, ceux-là externes à la question salariale proprement dite, ont aussi favorisé cette croissance continue des salaires depuis la fin de la Deuxième Guerre mondiale: la pénurie d'enseignants, et surtout d'enseignants qualifiés, la durée plus longue et l'amélioration de la formation du personnel, la présence de plus en plus grande des hommes - qui sont généralement plus scolarisés que les femmes et dotés d'un plus grand nombre d'années d'expérience, - ainsi que le renforcement des bases des associations syndicales.

Les conditions salariales du personnel enseignant laïque des secteurs protestant et catholique bénéficient d'un taux de croissance comparable, à l'exception de la première moitié des années 1960, où la croissance est plus accentuée dans ce dernier secteur (tableau 8). Ce sont d'ailleurs les salaires du personnel enseignant catholique qui ont le plus profité de la prospérité des années cinquante et soixante avec une augmentation allant, en moyenne, de $510 \%$ à $782 \%$ chez les enseignantes, entre 1941-1961 et 1961-1981, et de $179 \%$ à $416 \%$ chez les enseignants, au cours de la même période. Ce sont donc, en ordre décroissant, les enseignantes et enseignants laïques catholiques, suivis des enseignantes et des enseignants laïques protestants qui ont vu le plus augmenter leur salaire au cours de la période. Cette situation est principalement attribuable aux politiques de normalisation des dépenses des commissions scolaires et de rattrapage salarial, mises en place en 1965-1966 et consolidées par l'adoption en 1967 de la Loi 25. Ces politiques ont eu pour conséquence, à court terme, le maintien de l'augmentation salariale des enseignants protestants à un taux particulièrement faible tandis que l'augmentation des autres catégories d'enseignants enregistrait des hausses impressionnantes, ce qui devait aboutir, à plus long terme, à une équité salariale entre les différents membres de la profession enseignante du Québec.

Chez les enseignants du secteur protestant, certaines disparités salariales, enregistrées surtout dans la première moitié du $\mathrm{XX}^{\mathrm{e}}$ siècle, 
sont discernables selon le milieu géographique, le sexe ou le niveau d'enseignement. Le salaire moyen des enseignantes des écoles élémentaires rurales, par exemple, y est généralement de $26 \%$ à $45 \%$ inférieur à celui des enseignantes des écoles secondaires du même milieu et de $53 \%$ à $66 \%$ inférieur à celui des enseignantes des écoles élémentaires urbaines. D'importants écarts sont aussi à signaler chez les hommes, dont le salaire moyen, dans les écoles élémentaires rurales, est de $38 \%$ à $67 \%$ inférieur à celui des enseignants des écoles secondaires rurales, et de $57 \%$ à $84 \%$ inférieur à celui de leurs collègues masculins des écoles élémentaires situées en zone urbaine ${ }^{63}$.

Les disparités ont toutefois tendance à se résorber à partir du début des années 1950. L'écart salarial entre enseignants des milieux ruraux et urbains tend graduellement à se rétrécir, à la faveur de la généralisation de l'adoption de l'échelle salariale destinée aux commissions scolaires rurales. Le principe de la parité salariale entre hommes et femmes fait également son chemin, à partir de cette période, tandis que la nouvelle échelle salariale proposée en 1957 par la PAPT à ses membres et aux commissions scolaires de l'ensemble de la province ne s'appuie plus que sur deux paramètres: le niveau de formation des enseignants et leur nombre d'années d'expérience. Le sexe, le statut marital et le niveau d'enseignement, encore présents comme critères discriminatoires dans l'échelle salariale de 1952, sont abandonnés, ouvrant ainsi la porte à une uniformisation beaucoup plus étendue $^{64}$. De fait, contrairement à ce que N. Henchey affirme, dans son étude des réformes scolaires des années 1960, la première convention collective, signée à l'échelle provinciale en 1969, ne marque pas, du moins pas dans le secteur protestant, l'abolition des disparités salariales entre les sexes et les niveaux d'enseignement, mais vient plutôt conclure un processus enclenché depuis presque deux décennies ${ }^{65}$.

La centralisation des écoles élémentaires rurales ainsi que le regroupement administratif des commissions scolaires au sein de commissions scolaires centrales, depuis la deuxième moitié des années 1940, ont également favorisé ce mouvement d'uniformisation des salaires. D'une part, ils ont permis le regroupement d'un nombre plus grand d'enseignants à l'intérieur d'un même établissement, facilitant ainsi les échanges entre eux et le renforcement de leur solidarité, puis

63 Calculs établis sur les données du tableau 8.

64 Ibid., 94.

65 N. Henchey, «Quebec Education: the Unfinished Revolution», McGill Journal of Education, 7,2 (aut. 1972): 107. 
ils ont donné une plus grande stabilité économique aux employeurs et élargi leur territoire de négociation ${ }^{66}$.

\section{CONCLUSION}

L'analyse des données statistiques fait voir un portrait mouvant du corps enseignant œuvrant au sein des écoles du secteur protestant québécois au $\mathrm{XX}^{\mathrm{e}}$ siècle. Ce corps enseignant change tout au long de la période et s'adapte aux conjonctures socio-économiques, politiques et culturelles qui affectent le champ scolaire, en particulier, et la société, en général: exode rural, crises économiques, guerres, variations du taux de natalité et d'immigration, réformes scolaires diverses: des programmes de formation professionnelle, des structures administratives et des mécanismes de négociations.

Le corps enseignant se caractérise, sur le plan diachronique, par l'existence de deux générations: une première, qui remonte à la fin du $\mathrm{XIX}^{\mathrm{e}}$ siècle pour s'éteindre au cours des années quarante, et une seconde, s'échelonnant depuis les années cinquante jusqu'à nos jours. Si la première génération d'enseignants voit son nombre croître de façon importante, ainsi que ses conditions de travail et sa formation générale et professionnelle s'améliorer de façon significative, la seconde voit, pour sa part, les transformations de tout ordre se multiplier à un rythme impressionnant. De 1951 à 1981, par exemple, le nombre d'enseignants des écoles protestantes publiques augmente de plus de $127 \%$ tandis qu'entre 1965 et 1986 le nombre moyen d'années de scolarité de ces enseignants augmente de trois ans et leur salaire moyen, chez les hommes, de plus de $27000 \$(+380 \%)$, et chez les femmes, de plus de $24000 \$(+474 \%)$. Depuis le début du siècle jusqu'à aujourd'hui, à quelques exceptions près, c'est donc une croissance continue sur tous les fronts: croissance du nombre d'enseignants, durée accrue de la formation générale et professionnelle, resserrement des conditions d'émission des brevets d'enseignement et amélioration des conditions salariales.

66 Il faut cependant noter que les statistiques produites par le département de l'Instruction publique ont tendance, jusqu'au cours des années 1940, à surévaluer le montant du salaire moyen de l'ensemble du personnel enseignant puisqu'elles ne tiennent compte que des salaires des enseignants brevetés des écoles publiques et privées, ainsi qu'à sous-évaluer, au cours de la même période, le niveau salarial des enseignants de niveau secondaire puisqu'elles sont compilées selon le niveau d'enseignement des écoles et non selon celui des classes. Le nombre d'enseignants de classes élémentaires dans les high schools étant significatif, il y a lieu de croire qu'avant les années 1950 le salaire moyen des enseignants des classes secondaires était plus élevé que celui indiqué par les statistiques officielles. 
Deux éléments doivent également être mis en évidence. D'abord, l'indépendance relative de la croissance ou, du moins, du maintien du nombre des enseignants par rapport à celle de la clientèle scolaire, à partir des années 1960. Cette indépendance témoigne, entre autres, de l'émergence d'un corps enseignant mieux organisé, mieux structuré, capable de se donner des moyens d'action et d'intervention, rompant avec son rôle traditionnel d'acteur passif subissant la réalité scolaire plutôt que la dominant. La répartition sexuelle du personnel enseignant doit aussi être soulignée. Elle se caractérise par la part de plus en plus grande occupée par les hommes au sein de ce groupe, à la faveur, entre autres, du développement de l'enseignement secondaire, de l'amélioration des conditions salariales et de la hausse du prestige de la profession, ainsi que par la présence importante des femmes mariées sur le marché du travail enseignant, depuis le début des années quarante.

Ce portrait statistique du personnel enseignant nous donne une bonne idée de sa composition et de certaines de ses caractéristiques professionnelles, il constitue une base pour la poursuite de recherches sur la profession enseignante, son développement, son organisation, sa participation au champ scolaire. Une étude ultérieure, par exemple, sur les savoirs enseignants, ceux de la tradition et ceux des écoles de formation, sur la philosophie de l'éducation adoptée par les différents paliers de l'administration scolaire du secteur protestant et sur le syndicalisme en milieu scolaire permettrait sans doute de recouvrir l'armature quantitative ici élaborée d'un tissu de nouvelles informations, la complétant et la prolongeant. Il serait ainsi possible de développer une meilleure compréhension du rôle et des fonctions de l'enseignant dans la société québécoise, ainsi que de la perception qu'en ont les différents intervenants du monde scolaire. 